\title{
Higher-order Multi-dimensional Limiting Strategy for Correction Procedure via Reconstruction
}

\author{
Jin Seok Park*, Tae Kyu Chang* and Chongam Kim ${ }^{\dagger}$ \\ Department of Aerospace Engineering, Seoul National University, Seoul, 151-744, Korea
}

\begin{abstract}
Higher-order multi-dimensional limiting Process (MLP) [J. S. Park and C. Kim, Higherorder Multi-dimensional Limiting Strategy for Discontinuous Galerkin Methods in Compressible Inviscid and Viscous Flows, Comp. \& Fluids, In press] is improved and applied to correction procedure via reconstruction (CPR) on unstructured grids. MLP, which has been originally developed in finite volume method (FVM), provides an accurate, robust and efficient oscillation-control mechanism in multiple dimensions for linear reconstruction. This limiting philosophy can be hierarchically extended into higher-order $P n$ reconstruction. The resulting algorithms, called the hierarchical MLP, facilitate the accurate capturing of detailed flow structures in both continuous and discontinuous regions. This algorithm has been developed in the modal DG framework, but it also can be formulated into a nodal framework, most notably the CPR framework. Troubled-cells are detected by applying the MLP concept, and the final accuracy is determined by the projection procedure and MLP limiting step. Through extensive numerical analyses and computations, it is demonstrated that the proposed limiting approach yields the desired accuracy and outstanding performances in resolving compressible inviscid and viscous flow features.
\end{abstract}

\section{Introduction}

Up to now, second-order accurate computational fluid dynamics (CFD) methods with some discontinuitycapturing strategy are widely used to analyze compressible viscous flows. Theoretically, these approaches guarantee the minimal accuracy to recover essential physics of high-Reynolds number compressible flows. Indeed, they have witnessed remarkable successes in many classes of engineering and scientific applications. At the same time, however, they also unveil some limitations, particularly in capturing unsteady vortexdominated flow structures due to excessive numerical diffusion. From this perspective, higher-order methods are convincing alternatives in a sense that they can provide the detailed flow structures by upgrading the accuracy of spatial and temporal discretization with reasonable computational resources. ${ }^{1-3}$

In the last decade, various higher-order discretization methods have been developed. In order to handle complex geometry, these methods usually combine merits of both finite volume methods (FVM) and finite elements methods (FEM), which makes it possible to develop higher-order approximation for each cell within minimal stencil. Discontinuous Galerkin (DG) method is one of the widely-used and well-developed higherorder methods in hyperbolic conservation laws. Strong mathematical background and numerical analysis support it, but the formulations are rather complicated and expensive. Alternative approaches, such as spectral volume $(\mathrm{SV})^{4}$ and spectral difference $(\mathrm{SD})^{5}$ methods, are also studied to improve computational efficiency and numerical stability. Recently, Huynn proposed the flux reconstruction (FR) method, ${ }^{6,7}$ which provides an unifying framework of the above-mentioned methods (DG, SV and SD). Furthermore, Wang introduced a lifting collocation operator ${ }^{8}$ to deal with multi-dimensional problems on simplex and mixed grids. The two approaches, combined and renamed as correction procedures via reconstruction (CPR), share many merits of higher-order methods in terms of accuracy and stability, while it is more simple and efficient. Recent studies also show some encouraging results to resolve compressible flows. ${ }^{3}$

${ }^{*} \mathrm{Ph}$. D. candidate, Research Assistant, Department of Aerospace Engineering, Seoul National University, AIAA Student Member.

${ }^{\dagger}$ Professor, Department of Aerospace Engineering, Institute of Advanced Aerospace Technology, Seoul National University, AIAA Associate Fellow, Corresponding author: chongam@snu.ac.kr. 
However, there are a few obstacles to extend higher-order CFD methods, including CPR, into high speed unsteady flows. One of them is to design a robust and efficient oscillation-control algorithm to suppress unwanted oscillations around discontinuities without compromising the higher-order nature in smooth region. The main reason for such oscillations is the lack of providing a proper diffusion mechanism. Regarding this issue, two options are available. One is to add a properly tuned artificial viscosity term around shock region, ${ }^{9}$ which usually depends on flow structure and mesh system. The other resorts to some limiting algorithms which are mostly borrowed from finite volume methods.

Cockburn and Shu developed the first successful TVB-based limiter for the DG methods, ${ }^{10}$ which enforces the TVD/TVB condition in the mean sense by controlling the reconstructed slopes. This idea was generalized into the form of higher-order moments, ${ }^{11-14}$ and the improved methods have recently been studied as well. ${ }^{15,16}$ On the other hand, limiting strategies based on WENO-type reconstruction were also combined with higher-order DG method. ${ }^{17-19}$ While most of these limiting studies are formulated in DG framework, it is applicable to CPR and other higher-order methods. However, it has to be emphasized that the fundamental issue of limiting algorithms essentially lies in the the mathematical analyses based on the one-dimensional convection equation, which do not in general guarantee multi-dimensional monotonicity. Especially, the diminished numerical viscosity of higher-order CFD methods triggers multi-dimensional oscillations more often, and thus the robust and accurate shock-capturing philosophy for multi-dimensional flow is really necessary.

Recently, the multi-dimensional limiting process (MLP) has been successfully proposed in the FVM framework. Compared with traditional limiting strategies, such as the TVD or ENO-type limiting, the MLP limiting efficiently controls unwanted oscillations particularly in multi-dimensional flow situations. By imposing the MLP condition on both cell-averaged and cell-vertex values, the MLP limiting can efficiently follow the multi-dimensional flow physics, leading to the global/local $L^{\infty}$ stability. A series of previous researches ${ }^{20-22}$ demonstrated that the MLP limiting possesses superior characteristics in terms of accuracy, robustness and efficiency in inviscid and viscous computations on structured and unstructured grids within the FVM framework. Since the proposed limiting algorithm relies only on the MLP stencil regardless of the order of approximation, it facilitates an easy extension to popular higher-order methods, such as DG methods. As a way to stabilize the higher-order DG method, the original MLP condition is extended to take into account the behavior of local extrema produced by a cell-wise higher-order approximation. As a consequence, the augmented MLP condition and the MLP-based troubled-cell marker are obtained, which pave the way to obtain the hierarchical DG-MLP formulation. ${ }^{23}$

In this study, we extend the MLP limiting philosophy into the higher-order CPR to compute compressible inviscid and viscous flows. At first, the hierarchical MLP developed in DG framework, are implemented into the CPR method. Troubled-cells are marked by the augmented MLP condition and projection procedure to lower-order polynomial space, and the MLP slope limiting controls the degree of freedoms. By examining oscillatory behavior in sub-cell distribution and its propagation, P1-projected MLP condition is newly proposed and the improved hierarchical MLP strategy is developed. The present work is organized as follows. The baseline discretization methods are briefly summarized in Section II. Then, the hierarchical MLP limiting with the augmented MLP condition and P1-projected MLP condition is described in Section III and its implementation on both CPU and GPU. In Section IV, extensive numerical experiments are carried out to verify the favorable properties of the proposed limiting strategy for inviscid and viscous compressible flows. Finally, conclusions are given in Section V.

\section{Correction procedures via reconstruction}

In order to analyze the compressible flows, the following governing equations are considered.

$$
\frac{\partial \mathbf{Q}}{\partial t}+\nabla \cdot \mathbf{F}=0,
$$

where $\mathbf{Q}$ is the conservative variable vector and $\mathbf{F}$ is the flux vector.

The spatial discretization starts from the weak form of Eq. (1) on the cell $T_{j}$.

$$
\int_{T_{j}} \frac{\partial \mathbf{Q}}{\partial t} \mathbf{W} d V+\int_{\partial T_{j}} \mathbf{F} \cdot \mathbf{n} \mathbf{W} d S-\int_{T_{j}} \mathbf{F} \cdot \nabla \mathbf{W} d V=0,
$$

where $\mathbf{W}$ is a test function vector, and $\mathbf{n}$ is the outward unit normal vector. 
Distribution within a cell is then approximated in a suitably smooth function space $V^{n}$, which usually consists of polynomials of order up to $n$. After applying monotone numerical fluxes and integrating by part again, the approximated solution in $V^{n}$ on the cell $T_{j}$ can be written as follows.

$$
\int_{T_{j}} \frac{\partial \mathbf{Q}_{j}^{h}}{\partial t} \mathbf{W} d V+\int_{\partial T_{j}}\left(\mathbf{H}\left(\mathbf{Q}_{j k}^{h}, \mathbf{Q}_{k j}^{h}\right)-\mathbf{F}\left(\mathbf{Q}_{j k}^{h}\right)\right) \cdot \mathbf{n} \mathbf{W} d S+\int_{T_{j}} \nabla_{h} \cdot \mathbf{F}\left(\mathbf{Q}_{j}^{h}\right) \mathbf{W} d V=0,
$$

where $\mathbf{Q}_{j k}^{h}$ is the cell interface state vector in the direction from $T_{j}$ to its neighboring cell $T_{k}$, and $\mathbf{H}\left(\mathbf{Q}_{L}, \mathbf{Q}_{R}\right)$ is the tensors of a numerical flux function.

Using the lifting operator, the second integral term can be replaced by a correction field $\delta_{j} \in V^{n}$ and we can obtain following formulation.

$$
\int_{T_{j}}\left[\frac{\partial \mathbf{Q}_{j}^{h}}{\partial t}+\nabla_{h} \cdot \mathbf{F}\left(\mathbf{Q}_{j}^{h}\right)+\delta_{j}\right] \mathbf{W} d V=0 .
$$

By projecting the second term onto $V^{n}$, Eq. (4) with a proper test space can be simplified as follows.

$$
\frac{\partial \mathbf{Q}_{j}^{h}}{\partial t}+\Pi\left(\nabla_{h} \cdot \mathbf{F}\left(\mathbf{Q}_{j}^{h}\right)\right)+\delta_{j}=0,
$$

where $\Pi$ is the projection operator. For the linear conservation law, the projection operator can be skipped. In CPR method, higher-order approximation on each cell is approximated by solution points as

$$
\mathbf{Q}_{j}^{h}(\mathbf{x}, t)=\sum_{i}^{\text {ndof }} \mathbf{Q}_{i, j}^{h}(t) L_{i, j}(\mathbf{x})
$$

where $L_{i, j}(\mathbf{x})$ is the Lagrange polynomial and $\mathbf{Q}_{i, j}^{h}$ is the state vector at solution point $\mathbf{x}_{i}$ on the cell $T_{j}$. For each solution point, Eq. (5) is imposed as

$$
\begin{array}{r}
\frac{\partial \mathbf{Q}_{i, j}^{h}}{\partial t}+\Pi_{i}\left(\nabla_{h} \cdot \mathbf{F}\left(\mathbf{Q}_{j}^{h}\right)\right)+\delta_{i, j}=0, \\
\delta_{i, j}=\frac{1}{\left|T_{j}\right|} \sum_{f=e_{j k} \in T_{j}} \sum_{l} \alpha_{l, f, j}[\mathbf{F}]_{l, f} \cdot \mathbf{n}\left|e_{j k}\right|,
\end{array}
$$

where

$$
[\mathbf{F}]_{l, f}=\mathbf{H}\left(\mathbf{Q}_{l, j k}^{h}, \mathbf{Q}_{l, k j}^{h}\right)-\mathbf{F}\left(\mathbf{Q}_{l, j k}^{h}\right) .
$$

Here, $\mathbf{Q}_{l, j k}^{h}$ is the approximated value at $l$-th solution point on face $e_{j k}$. The details of CPR, such as solution points and $\alpha_{l, f, j}$, can be found in Ref. ${ }^{8}$

To handle the second term in Eq. (7) for non-linear flux, there are two approaches; Lagrange polynomial (LP) and Chain Rule (CR). Both become problematic in solving non-linear equations, such as aliasing error for LP and conservation error for CR. Recently, an efficient cure for the conservation issue is devised ${ }^{24}$ and the present development is based on the conservative CPR method.

The viscous stress and heat flux are treated with an auxiliary variable vector,

$$
\Theta-\nabla \mathbf{Q}=\mathbf{0} .
$$

It is also discretized in CPR method.

$$
\Theta_{i, j}=\left(\nabla \mathbf{Q}_{i, j}^{h}\right)+\frac{1}{\left|T_{j}\right|} \sum_{f=e_{j k} \in T_{j}} \sum_{l} \alpha_{l, f, j}\left[\left(\mathbf{Q}_{l, j k}^{c o m}-\mathbf{Q}_{l, j k}^{h}\right]_{l, f} \mathbf{n}\left|e_{j k}\right| .\right.
$$

Some viscous flux schemes for DG method can be applied to CPR as well, ${ }^{25}$ and BR2 scheme ${ }^{26}$ is used in this work.

$$
\begin{aligned}
\mathbf{Q}_{l, j k}^{c o m} & =\frac{1}{2}\left(\mathbf{Q}_{l, j k}^{h}+\mathbf{Q}_{l, k j}^{h},\right) \\
\nabla \mathbf{Q}_{l, j k}^{c o m} & =\frac{1}{2}\left(\nabla \mathbf{Q}_{l, j k}^{h}+\mathbf{r}_{l, j k}+\nabla \mathbf{Q}_{l, k j}^{h}+\mathbf{r}_{l, k j}\right) .
\end{aligned}
$$


Here, $\mathbf{r}_{l, j k}$ reflects the jump of the conservative variable at the face $e_{j k}$.

$$
\mathbf{r}_{l, j k}=\sum_{l} \alpha_{l, f, j}\left[\left(\mathbf{Q}_{l, j k}^{c o m}-\mathbf{Q}_{l, j k}^{h}\right]_{l, f} \mathbf{n}\left|e_{j k}\right| .\right.
$$

For time integration, nonlinear stable methods are applied, such as the third-order accurate TVD Runge-Kutta method or the five-stage fourth-order accurate strong stability preserving Runge-Kutta method $(\operatorname{SSPRK}(5,4)) .{ }^{27}$ The following definition of time step is used.

$$
\Delta t=\frac{C F L}{2 n+1} \frac{h}{\left|\lambda_{c}^{\max }\right|+d\left|\lambda_{v}^{\max }\right| \frac{2 n+1}{h}},
$$

where $h$ is the radius of inscribed circle for triangular element. $\lambda_{c}^{\max }$ and $\lambda_{v}^{\max }$ are the maximum wave speed of inviscid and viscous flux, respectively. CFL number has been set to 0.9 for CPR-P1 or P2 with third-order TVD Runge-Kutta method, and 1.4 for CPR-P3 with $\operatorname{SSPRK}(5,4)$.

\section{Higher-order Multi-dimensional Limiting Strategy for CPR}

As well as stable time integration methods, the proper oscillation-control mechanism is essential to resolve compressible flows, especially the flows involved with shock waves. Limiting should be activated only on the troubled-cells to maintain higher-order accuracy across smooth extrema. An accurate troubled-cell marker, followed by a sophisticated limiting, is thus crucial to obtain an accurate monotone profile in the higherorder approximation. Some troubled-cell markers, such as the TVB marker ${ }^{10}$ or KXRCF marker, ${ }^{28}$ have been developed and combined with slope limiters or WENO-type limiters, but the precise detection of the troubled-cells is not an easy task. We propose two new indicators based on the MLP concept to detect the troubled-cells. At first, we briefly summarize MLP-u slope limiters and describe the implementation of the hierarchical MLP for CPR. The last subsection deals with an improved troubled-cell marker with the $P 1$-projected MLP condition and limiting strategy.

\section{A. MLP-u Slope Limiter}

In order to enforce multi-dimensional monotonicity, the MLP condition has been proposed in the finite volume methods. This condition is an extension of the one-dimensional monotonicity condition, by considering the case where the direction of local flow gradient is not aligned to the local grid line. The starting point of the MLP condition is that local extrema always occur at vertex point when sub-cell distribution is linear. This observation manifests that, i) treatment of vertex point is essential in limiting stage, ii) all information around vertex point should be incorporated to avoid multi-dimensional oscillations, which leads to the following MLP condition.

$$
\bar{q}_{v_{i}}^{\min } \leq q\left(\mathbf{x}_{v_{i}}\right) \leq \bar{q}_{v_{i}}^{\max }
$$

with

$$
\bar{q}_{v_{i}}^{\min }=\min _{T_{k} \in S_{v_{i}}}\left(\bar{q}_{k}\right), \quad \bar{q}_{v_{i}}^{\max }=\max _{T_{k} \in S_{v_{i}}}\left(\bar{q}_{k}\right) .
$$

Here, $\bar{q}_{k}$ is the cell-averaged value on $T_{k}$ and $\mathbf{x}_{v_{i}}$ is the position vector at the vertex $v_{i}$. Two stencil $S_{v_{i}}$ and $S_{T_{j}}$ are defined by

$$
S_{v_{i}}=\left\{T_{k} \mid v_{i} \in T_{k} \text { for some } v_{i}\right\}, \quad S_{T_{j}}=\left\{T_{k} \mid v_{i} \in T_{k}, \text { for all } v_{i} \in T_{j}\right\} .
$$

In other words, $S_{v_{i}}$ is the union of computational cells sharing the vertex $v_{i}$ and $S_{T_{j}}$ is the union of computational cells sharing any vertex of cell $T_{j} . S_{T_{j}}$ is called the MLP stencil. ${ }^{21,22}$ It is noted that the MLP condition can be applied to any type of mesh since it does not assume particular mesh connectivity. At the same time, it is also observed that well-controlled vertex value at interpolation/limiting stage makes it possible to produce monotonic distribution of cell-averaged values. Extensive numerical experiments ${ }^{20-22}$ strongly support that full realization of Eq. (16) is very effective to preserve accurate monotone profiles.

This philosophy can be readily extended on unstructured grids with second-order accurate reconstruction. Sub-cell interpolation may start from the unstructured version of the MUSCL-type linear reconstruction as follows.

$$
q_{j}(\mathbf{x})=\bar{q}_{j}+\phi_{j} \nabla \bar{q}_{j} \cdot\left(\mathbf{x}-\overline{\mathbf{x}}_{j}\right)
$$


where $q$ is the the state variable, $\nabla \bar{q}_{j}$ is the gradient within the cell $T_{j}$. $\mathbf{x}$ is the position vector and $\overline{\mathbf{x}}_{j}$ is the the centroid of the cell $T_{j}$. After applying the MLP condition to vertex point, the MLP slope limiter is introduced to ensure multi-dimensional monotonicity by considering all the distributions around the common vertex $v_{i}$. The range of the MLP slope limiting is then obtained as follows.

$$
0 \leq \phi_{j} \leq \max \left(\frac{\bar{q}_{v_{i}}^{\min }-\bar{q}_{j}}{\nabla \bar{q} \cdot\left(\mathbf{x}_{v_{i}}-\overline{\mathbf{x}}_{j}\right)}, \frac{\bar{q}_{v_{i}}^{\max }-\bar{q}_{j}}{\nabla \bar{q} \cdot\left(\mathbf{x}_{v_{i}}-\overline{\mathbf{x}}_{j}\right)}\right)
$$

From Eq. (20), the MLP-u slope limiters can be obtained as follows.

$$
\phi_{M L P}=\min _{\forall v_{i} \in T_{j}} \begin{cases}\Phi\left(r_{v_{i}, j}\right) & \text { if } \nabla \bar{q}_{j} \cdot \mathbf{r}_{v_{i}, j} \neq 0 \\ 1 & \text { otherwise }\end{cases}
$$

where

$$
r_{v_{i}, j}=\max \left(\frac{\bar{q}_{v_{i}}^{\min }-\bar{q}_{j}}{\nabla \bar{q}_{j} \cdot\left(\mathbf{x}_{v_{i}}-\overline{\mathbf{x}}_{j}\right)}, \frac{\bar{q}_{v_{i}}^{\max }-\bar{q}_{j}}{\nabla \bar{q}_{j} \cdot\left(\mathbf{x}_{v_{i}}-\overline{\mathbf{x}}_{j}\right)}\right) .
$$

By determining $\Phi(r)$ to satisfy the maximum principle, we have the MLP-u1 and MLP-u2 limiters. Detailed implementation in unsteady and steady flows can be found in Refs. ${ }^{21,22}$

The MLP limiting is supported by the maximum principle in ensuring the monotonicity in multiple dimensions. For multi-dimensional scalar conservation law, the MLP limiting guarantees the following local maximum principle under a suitable CFL condition. ${ }^{21,22}$

$$
\text { If } \min _{T_{k} \in S_{T_{j}}}\left(\bar{q}_{k}^{n}\right) \leq \bar{q}_{j}^{n} \leq \max _{T_{k} \in S_{T_{j}}}\left(\bar{q}_{k}^{n}\right) \text {, then } \min _{T_{k} \in S_{T_{j}}}\left(\bar{q}_{k}^{n}\right) \leq \bar{q}_{j}^{n+1} \leq \max _{T_{k} \in S_{T_{j}}}\left(\bar{q}_{k}^{n}\right) .
$$

In conjunction with the MLP condition (Eq. (16)), Eq. (23) simply states that the MLP limiting satisfies the MLP condition at cell-averaged value at every time step.

The MLP condition on the MLP stencil makes it realizable to capture multi-dimensional flow structure accurately while maintaining the required order-of-accuracy. From Eqs. (9) and (13), the updated solution by the MLP limiting satisfies the maximum principle both on cell-averaged and cell-vertex values, though the stencil involved is a bit different. In addition, from the global/local $L^{\infty}$ stability of computed solutions, the MLP limiting satisfies the LED condition in a truly multi-dimensional way. ${ }^{22}$

\section{B. Augmented MLP Condition}

The MLP condition was used to identify and control the maximum-principle-violating cells in second-order finite volume methods. ${ }^{21,22}$ For higher-order polynomial approximations greater than $P 1$ approximation, however, additional condition is necessary because the P1-based MLP condition may omit some troubled cells that violate the maximum principle.

If we assume a discontinuity near vertex, as shown in Fig. 1, higher-order $P n$ approximation would trigger unwanted oscillation in the blue-shaded region. For $P 1$ approximation, the approximated value at any quadrature point in $T_{j}$ can be readily controlled by suitably limiting the vertex values at which the extrema always occur. For greater than $P 1$ approximation, we may have a quadrature point at which the approximated value can lie outside the range imposed by the MLP condition (Eq. (16)), thus incurring the danger of violating the maximum principle (Eq. (23)). This may occur even if the vertex value does satisfy the MLP condition. The MLP condition imposed on a single cell $T_{j}$ is not complete enough to handle higher-order distribution and it may allow spurious oscillation for the situation in Fig.1. As a remedy, we require all approximated vertex values for $S_{v_{i}}$ to satisfy the MLP condition. In other words, when we check whether $T_{j}$ is a troubled cell or not, we impose the MLP condition on $S_{v_{i}}$ not on $T_{j}$. From this perspective, we propose a stricter condition, termed the augmented MLP condition, as follows. The augmented MLP condition is then used as the MLP-based troubled-cell marker for higher-order approximation.

$$
\bar{q}_{v_{i}}^{\min } \leq \min _{T_{k} \in S_{v_{i}}}\left(q_{k}^{h}\left(\mathbf{x}_{v_{i}}\right)\right), \max _{T_{k} \in S_{v_{i}}}\left(q_{k}^{h}\left(\mathbf{x}_{v_{i}}\right)\right) \leq \bar{q}_{v_{i}}^{\max } .
$$

If any $q_{k}^{h}\left(\mathbf{x}_{v_{i}}\right)$ violates Eq.(24), $T_{k} \in S_{v_{i}}$ is tagged as a troubled cell. The augmented MLP (or A-MLP) condition is consistent with the MLP condition for $P 1$ approximation. Numerical experiments strongly support that the MLP slope limiter with the A-MLP condition is quite successful in handling multi-dimensional oscillations. 


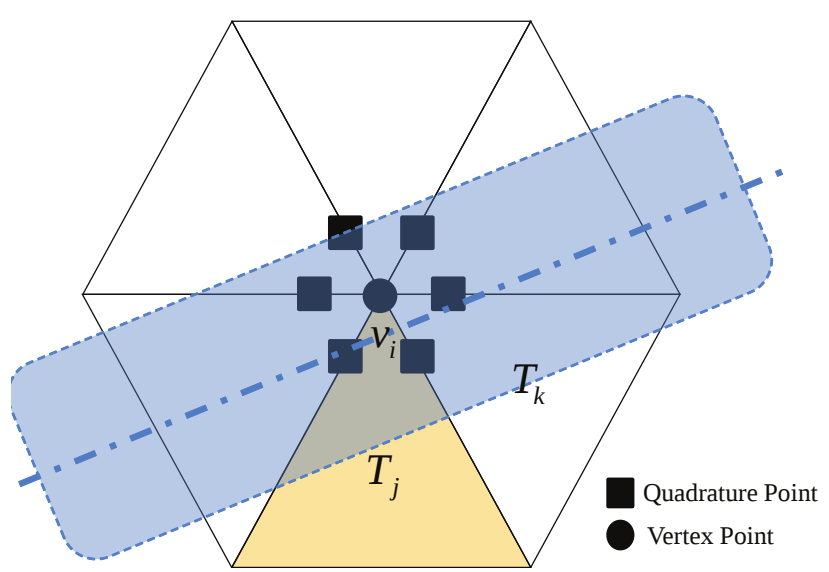

Figure 1: Discontinuity on the MLP stencil. (Dash-dot line is discontinuity and spurious oscillations may occur in the blue-shaded region.)

\section{Hierarchical MLP Limiting for CPR method}

Similar to the MLP condition, the augmented MLP condition itself does not provide any mechanism to distinguish local extrema. In order to preserve the accuracy across smooth extrema, a simple but effective extrema detector is introduced as follows.

$$
\Delta \bar{q}_{v_{i}}=\bar{q}_{v_{i}}^{\max }-\bar{q}_{v_{i}}^{\min } \leq K \Delta x^{2},
$$

where $K$ is a parameter to be determined.

The MLP-based troubled-cell marker given by the augmented MLP condition and the simple extrema detector results in quite successful performance to control multi-dimensional oscillations. In fact, the augmented MLP condition can be extended to general $P n$ approximation as an accurate troubled-cell marker. However, a snag remains when attempting to determine the optimal value of $K$ for the simple extrema detector. More seriously, the optimal value of $K$ may change depending on the order of polynomial approximation. Thus, the simple extrema detector is no longer employed, and instead, a hierarchical extrema detector for arbitrary $P n$ approximation is deduced by examining the behavior of local extrema around the vertex $v_{i}$ of the cell $T_{j}$.

First, we decompose the $P n$ approximation into the linear part ( $P n$-projected slope) and the higher-order part ( $P 1$-filtered $P n)$, as follows:

$$
q_{j}^{h, P n}\left(\mathbf{x}_{v_{i}}\right)=\bar{q}_{j}+\underbrace{\left(\Upsilon\left(\mathbf{x}_{v_{i}}\right)-\bar{q}_{j}\right)}_{P n \text {-projected slope }}+\underbrace{\left(q_{j}^{h, P n}\left(\mathbf{x}_{v_{i}}\right)-\Upsilon\left(\mathbf{x}_{v_{i}}\right)\right)}_{P 1 \text {-filtered } P n} .
$$

Here,

$$
\Upsilon\left(\mathbf{x}_{v_{i}}\right)=\Pi^{1} q_{j}^{h, P n}\left(\mathbf{x}_{v_{i}}\right) .
$$

The starting point is that the $P n$-projected slope can be interpreted as the average slope for the $P n$ approximation. Then, from the mean value theorem, if local extrema appear in the small neighborhood of the vertex $v_{i}$, the gradient direction of $q_{j}^{h, P n}\left(\mathbf{x}_{v_{i}}\right)$ could be substantially different from the direction of the $P n$-projected slope. If one goes up, the other would go down. Even if they show the same trend, the magnitude of the gradient of $q_{j}^{h, P n}\left(\mathbf{x}_{v_{i}}\right)$ would be smaller than that of the $P n$-projected slope. From this observation, we can deduce the following smooth extrema detector near the vertex $v_{i}$.

- $\mathbb{C} 1$. If there is a local maximum near the vertex $v_{i}$,

$P n$-projected slope $>0, P 1$-filtered $P n<0, q_{j}^{h, P n}\left(\mathbf{x}_{v_{i}}\right)>\bar{q}_{v_{i}}^{\min }$.

- $\mathbb{C} 2$. If there is a local minimum near the vertex $v_{i}$,

$P n$-projected slope $<0, P 1$-filtered $P n>0, q_{j}^{h, P n}\left(\mathbf{x}_{v_{i}}\right)<\bar{q}_{v_{i}}^{\max }$. 
The last inequalities $\left(q_{j}^{h, P n}\left(\mathbf{x}_{v_{i}}\right)>\bar{q}_{v_{i}}^{\min }, q_{j}^{h, P n}\left(\mathbf{x}_{v_{i}}\right)<\bar{q}_{v_{i}}^{\max }\right)$ are necessary to treat a stiff gradient which may include physical discontinuities. In order to deal with a nearly constant region particularly when local grids become very coarse, we add the following deactivation threshold.

$$
\left|q_{j}^{h, P n}\left(\mathbf{x}_{v_{i}}\right)-\bar{q}_{j}\right| \leq \max \left(\epsilon \times\left|\bar{q}_{j}\right|,\left|T_{j}\right|\right),
$$

where $\epsilon$ is a small number to distinguish a constant region with machine error, $1 \times 10^{-3}$ is a reasonable choice. $\left|T_{j}\right|$ is the area of cell $T_{j}$. Most of the extrema are well recognized by the condition $\mathbb{C} 1$ and $\mathbb{C} 2$, and only a very few cells are detected by Eq. (29).

By combining the A-MLP condition (Eq. (24)) and the extrema detector (Eq. (28) with Eq. (29)), we formulate the hierarchical MLP limiting scheme for arbitrary $P n$ approximation. The limiting procedure higher than $P 2$ approximation can be written as follows.

$$
\begin{aligned}
q_{j}^{h, p 2}(\mathbf{x}) & =\bar{q}_{j}+\phi_{M L P}\left(P 1_{j}(\mathbf{x})\right)+\varphi_{j}^{P 2}\left(P 2_{j}(\mathbf{x})\right) \\
q_{j}^{h, P 3}(\mathbf{x}) & =\bar{q}_{j}+\phi_{M L P}\left(P 1_{j}(\mathbf{x})\right)+\varphi_{j}^{P 2}\left(P 2_{j}(\mathbf{x})+\varphi_{j}^{P 3}\left(P 3_{j}(\mathbf{x})\right)\right), \\
\ldots & \\
q_{j}^{h, P n}(\mathbf{x}) & =\bar{q}_{j}+\phi_{M L P}\left(P 1_{j}(\mathbf{x})\right) \\
& +\varphi_{j}^{P 2}\left(P 2_{j}(\mathbf{x})+\varphi_{j}^{P 3}\left(P 3_{j}(\mathbf{x})+\varphi_{j}^{P 4}\left(\ldots+\varphi_{j}^{P n} P n_{j}(\mathbf{x})\right)\right)\right) .
\end{aligned}
$$

Here,

$$
P m_{j}(\mathbf{x})=\Pi^{m} q_{j}^{h, P n}(\mathbf{x})-\Pi^{m-1} q_{j}^{h, P n}(\mathbf{x}) .
$$

$\varphi_{j}^{P n}$ is the hierarchical MLP troubled-cell marker for the $P n$ approximation, which is formulated as follows:

$$
\varphi^{P n}=\min _{\forall v_{i} \in T_{j}}\left(\psi_{v_{i}, j}^{P n}\right)
$$

$$
\psi_{v_{i}, j}^{P n}= \begin{cases}1 & \text { if Eq. (24) or Eq. (28) with Eq. (29) is satisfied, } \\ 0 & \text { else. }\end{cases}
$$

The MLP limiting procedure of Eq. (30) can be applied in a hierarchical manner from a higher-order $P n_{j}$ mode to the lowest $P 2_{j}$ mode. The implementation procedure can be summarized as follows:

1. Examine the augmented MLP condition (Eq. (24)) with the $P n$ approximated solution at every vertex $v_{i}$ of the cell $T_{j}$.

2. Compute the hierarchical troubled-cell marker $\varphi_{j}^{P n}$ (Eq. (32)).

3. If the cell $T_{j}$ is tagged as a normal cell, the highest order term is kept unlimited and the limiting procedure is completed. Otherwise,

(a) if $n>2$, project the entire polynomial approximation onto $V^{n-1}$ space and obtain $P(n-1)_{j}(\mathbf{x})$. And, apply the A-MLP condition and repeat Step 2 for $P(n-1)$ approximation.

(b) if $n=2$, project the entire polynomial approximation onto $V^{1}$ space. $P 1_{j}(\mathbf{x})$ is limited by the MLP-u slope limiters (Eq. (21)).

In modal DG with orthogonal shape basis, above projection procedure is realized by simply truncating higher-order $P n$ mode. Since CPR is a nodal formulation, the projection procedure is carried out by obtaining $q_{j}^{h, P m}(\mathbf{x})$ from $P n$ approximation $(m \leq n)$.

$$
\sum_{l}\left(\int_{T_{j}} L_{l, j}^{P m}(\mathbf{x}) L_{k, j}^{P m}(\mathbf{x}) d V\right) \tilde{q}_{l, j}^{h, P m}=\int_{T_{j}} q_{j}^{h, P n}(\mathbf{x}) L_{k, j}^{P m}(\mathbf{x}) d V,
$$

where $L_{i, j}^{P n}(\mathbf{x})$ are the $n$-th order Lagrange polynomial for the solution point $\mathbf{x}_{i}$ on the cell $T_{j}$ for $P n$ approximation. Using this procedure, the hierarchical MLP can be implemented as follows. After checking 
the troubled-cell marker for $P n$ approximation, we obtain $P n-1$ projected polynomial via Eq. (34) for the troubled-cell $T_{j}$. Then, approximation at solution points $q_{i, j}^{h}$ is obtained.

$$
\Pi^{m} q_{j}^{h, P n}(\mathbf{x})=\sum_{l} \tilde{q}_{l, j}^{h, P m} L_{i, j}^{P m}(\mathbf{x}) .
$$

Thus we can remove $P n_{j}$ mode and this procedure is performed in a hierarchical manner until we finally apply MLP-u slope limiter when $P 2_{j}$ mode is limited. Figure 2 shows the schematic summary of the hierarchical MLP limiting procedure for CPR method.

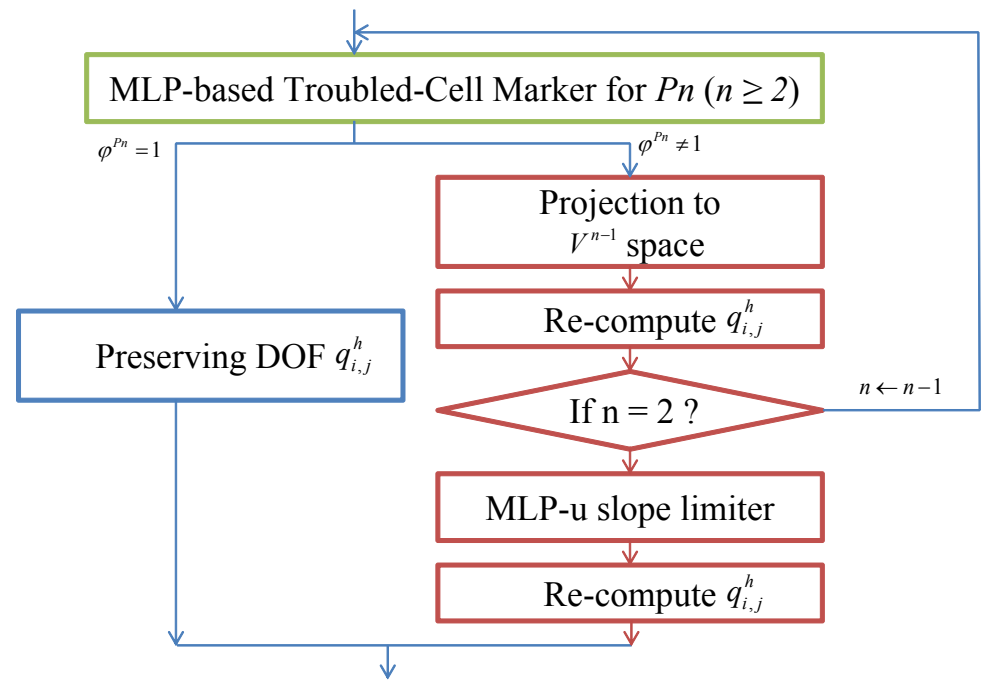

Figure 2: Flowchart of the MLP limiting procedure up to CPR-P2 reconstruction.

\section{P1-projected MLP Condition}

While the hierarchical MLP is successful to control multi-dimensional oscillations for higher-order methods, its performance to distinguish normal cells needs to be improved. The A-MLP condition is robust but appears to be too severe. To improve this situation, a MLP-based troubled-cell marker is newly proposed by examining the sub-cell distribution of Gibbs phenomena. For convenience, the analysis is performed in modal DG method but its consequence is also valid for CPR method.

In order to analyze behavior across discontinuous profile, we consider one-dimensional scalar conservation law with a simple discontinuous initial data.

$$
q_{t}+q_{x}=0, \quad q_{0}(x)=\left\{\begin{array}{ll}
1 & 0.25<x<0.75 \\
0 & \text { else }
\end{array} .\right.
$$

Computational domain consists of 80 cells on [0,1]. Figure 3 shows the sub-cell distribution after a few iterations with DG- $P 2$ and DG- $P 3$ approximation. Black, blue and red lines are $P n$ approximation $\left(q_{j}^{h, P n}(\mathbf{x})\right)$, $P 1$-projected approximation $\left(\Pi^{1} q_{j}^{h, P n}(\mathbf{x})\right)$ and cell-aveaged value $\left(\bar{q}_{j}\right)$, respectively. Circle denotes the end values of $P n$ approximation at each element. Although cell-averaged values maintain a monotonic profile, oscillations are already developed in the higher-order modes which are propagated into the cell-averaged values. When these oscillations start, $P 1$-projected approximation develops a steep gradient, warning the non-monotone distribution.

Figure 4 shows the sub-cell distribution of the Burgers' equation at $t=0.3$. The computational domain is the same and the initial condition is a sine wave.

$$
q_{0}(x)=\sin (2 \pi x) .
$$

Lines and symbol are the same as Fig. 3. As shock discontinuity is developed, oscillations start in $P n$ approximations and, at the same time, steep gradients of the projected- $P 1$ approximations can be observed. 


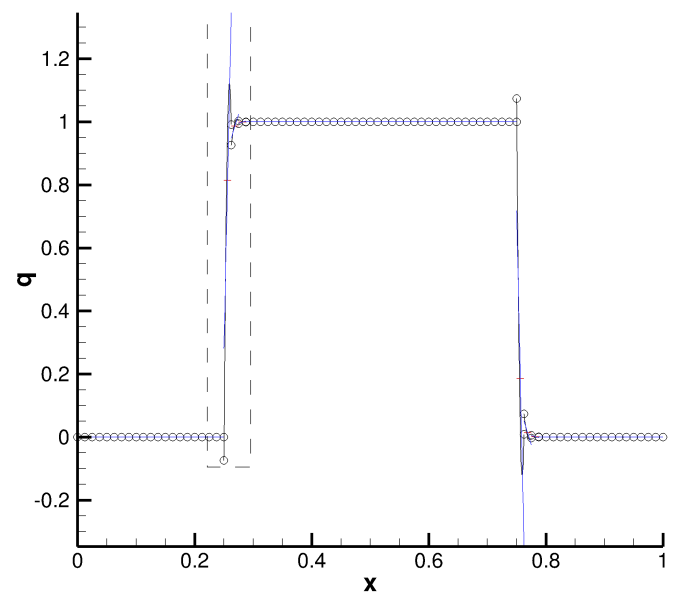

(a) DG-P2

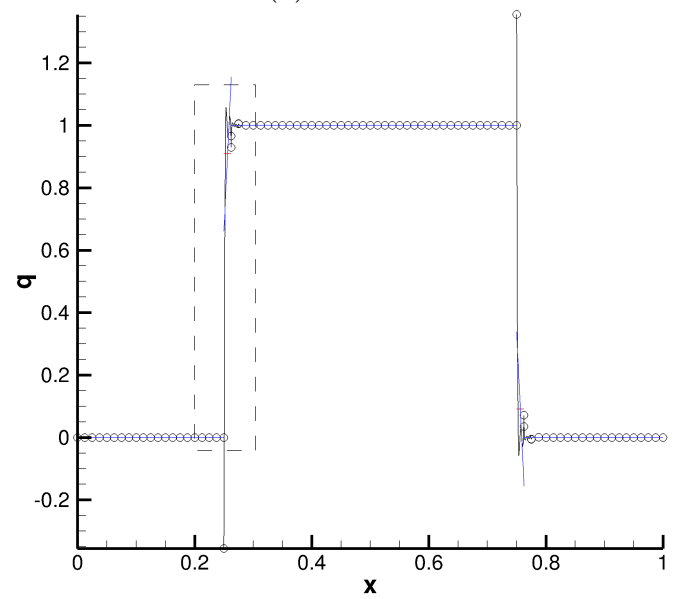

(c) DG-P5

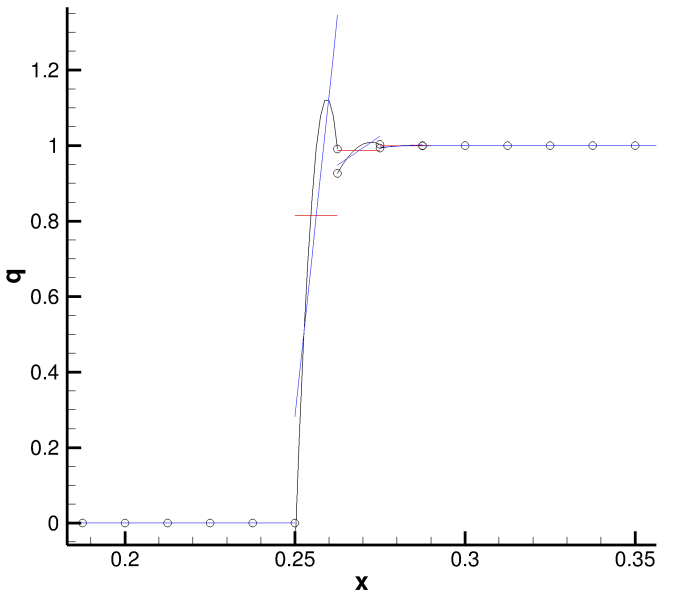

(b) DG-P2, closeup

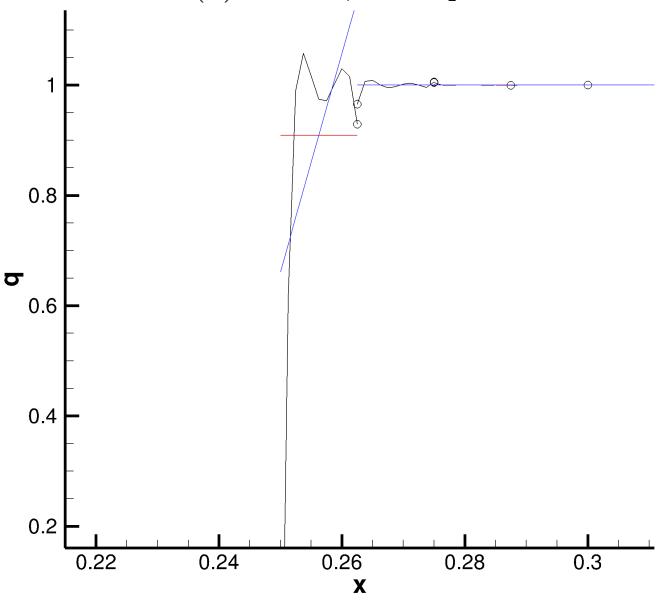

(d) DG-P5, closeup

Figure 3: Sub-cell distribution of square wave after a few iterations 


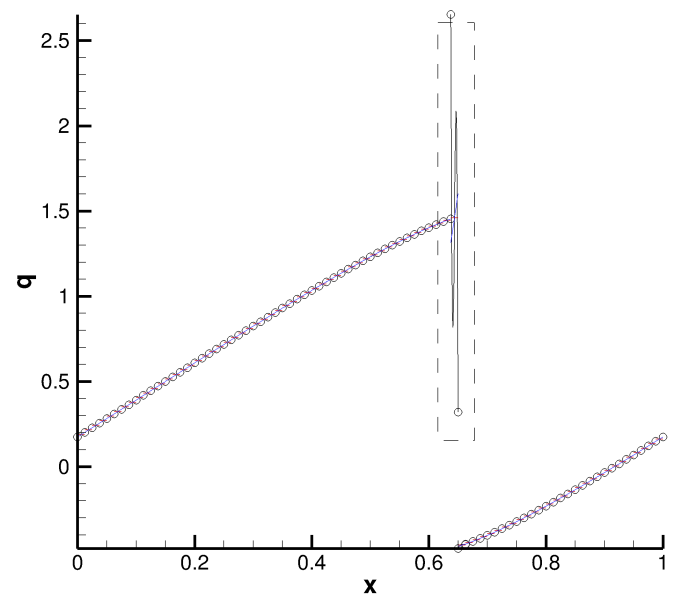

(a) DG-P3

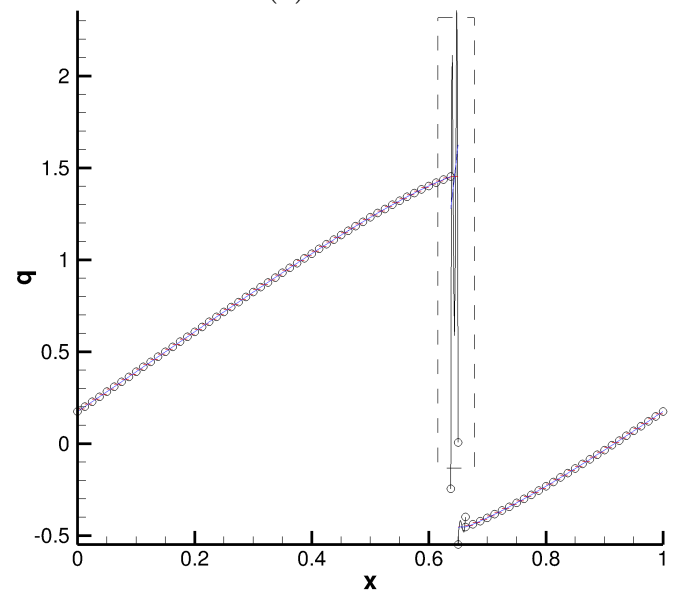

(c) DG-P4

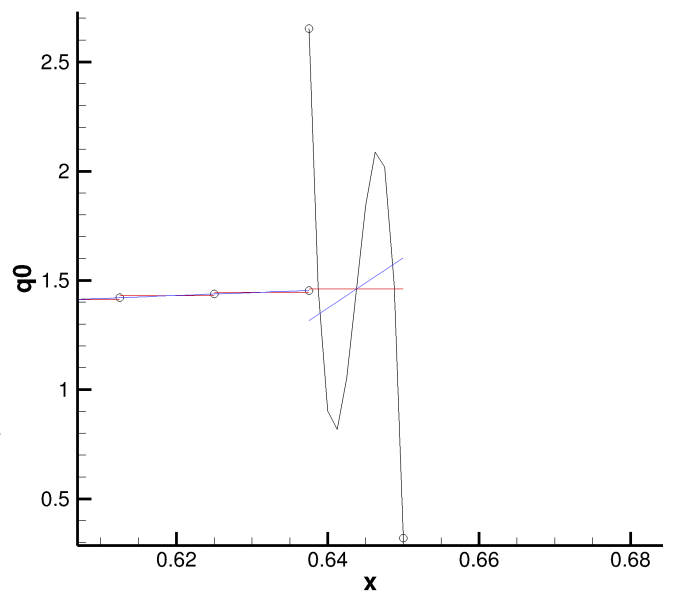

(b) DG-P3, closeup

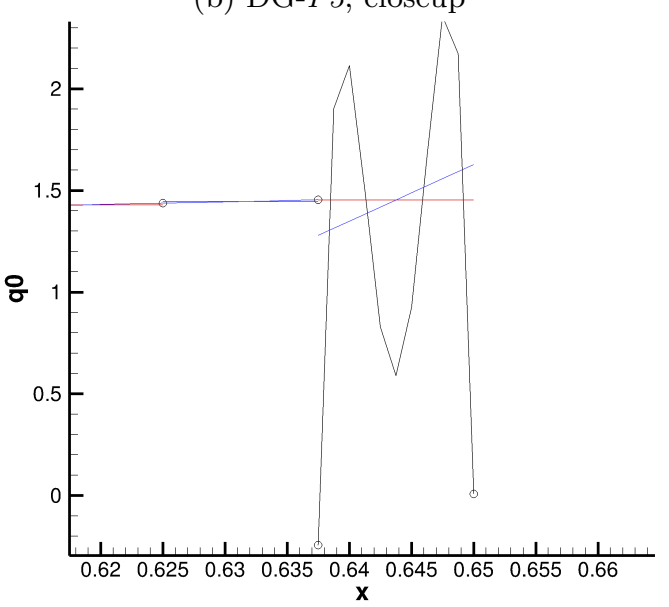

(d) DG-P4, closeup

Figure 4: Sub-cell distribution of Burgers' equation at $t=0.3$. 
From this result, it is observed that oscillations are propagated from $\mathrm{Pn}$ sub-cell distribution to cellaveraged value. In other words, oscillations in sub-cell distribution strongly affects the $P 1$-projected term, indicating that examining the monotonicity of the $P$ 1-projected term is enough to detect oscillations in the higher-order modes. Based on this observation, we newly propose a limiting condition for higher-order approximation.

$$
\min \left(\bar{q}_{i}, \bar{q}_{i+1}\right) \leq \Pi^{1} q_{j}^{h, P n}\left(x_{i+1 / 2}\right) \leq \max \left(\bar{q}_{i}, \bar{q}_{i+1}\right) .
$$

It is noted that this condition is derived from the linear term, and thus it is readily extended into multiple dimensions with the MLP philosophy, leading to the following P1-projected MLP condition.

$$
\bar{q}_{v_{i}}^{\min } \leq \Pi^{1} q_{j}^{h, P n}\left(\mathbf{x}_{v_{i}}\right) \leq \bar{q}_{v_{i}}^{\max } .
$$

To avoid accuracy loss across the smooth extrema, the same extrema detector of the hierarchical MLP (Eqs. (28) and (29)) is used. Again, the resulting limiting strategy is applied in a hierarchical manner (Eq. (30)). The MLP troubled-cell marker $\varphi_{j}^{P n}$ (Eq. (32)) is similarly defined by

$$
\psi_{v_{i}, j}^{P n}=\left\{\begin{array}{ll}
1 & \text { if Eq. (39) or Eq. (28) with Eq. (29) is satisfied } \\
0 & \text { else }
\end{array} .\right.
$$

The overall limiting procedure can be summarized as follows.

1. Obtain $q_{j}^{h, P n}(\mathbf{x})$ and examine the $P 1$-projected MLP condition on the cell $T_{j}$.

2. For $P n$ approximation, check the extrema detector (Eq. (28) with Eq. (29)) and compute the troubledcell marker $\varphi_{j}^{P n}$ (Eq. (32)) with $\psi_{v_{i}, j}^{P n}$ (Eq. (40)).

3. If the cell $T_{j}$ is tagged as a normal cell, the highest order term is kept unlimited and the limiting procedure is completed. Otherwise,

(a) if $n>2$, project the entire polynomial approximation onto $V^{n-1}$ space and obtain $P(n-1)_{j}(\mathbf{x})$. And, repeat Step 2 for $P(n-1)$ approximation.

(b) if $n=2$, project the entire polynomial approximation onto $V^{1}$ space. $P 1_{j}(\mathbf{x})$ is limited by the MLP-u slope limiters (Eq. (21)).

Figures 5 and 6 show the same one-dimensional linear wave equation and Burgers' equation with the $P 1$ projected limiting condition at $t=1.0$ and $t=0.3$, respectively. Oscillations are effectively suppressed and cell-averaged values maintain monotonicity. Slight oscillations may be observed in sub-cell distribution of $P n$ approximation, but sub-cell oscillation is well controlled within the range of extrema detection condition and do not affect on cell-averaged values. For multi-dimensional case, similar results can be obtained and it will be discussed in the next section.

\section{E. Extension to Flow System}

The troubled-cell markers and slope limiters for system of equations should reflect the flow physics. Density or entropy variable is used for the MLP troubled-cell marker to identify physical discontinuities. The marker by entropy variable is slightly more robust, especially resolving very strong shock or nearly vacuum state. In this study, all experiments carried out with MLP troubled-cell marker by density variation. As in the case of FVM, the MLP limiting is applied to conservative variables since characteristic decomposition is not essential. Negative pressure and/or density at solution points, if any, is treated by employing a simple scaling technique. ${ }^{15}$

\section{F. Parallelization}

While higher-order methods remarkably enhance the solution accuracy, all degree of freedoms should be handled spatially and temporally, leading to a significant increase of computational cost per cell. In order to relieve computational burden, parallelization techniques are employed in both CPU and GPU computing environments. Grid partitioning by the METIS library ${ }^{29}$ and parallelization with the MPI standard are implemented for CPU programming. 


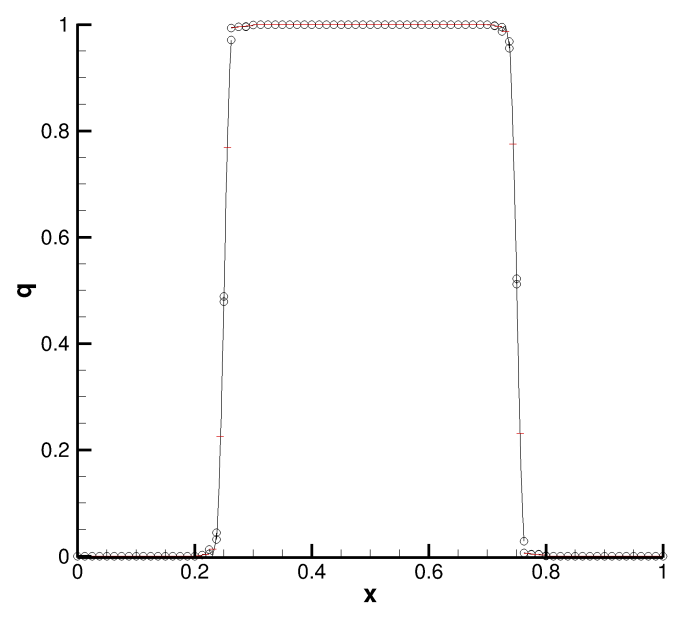

(a) DG-P2

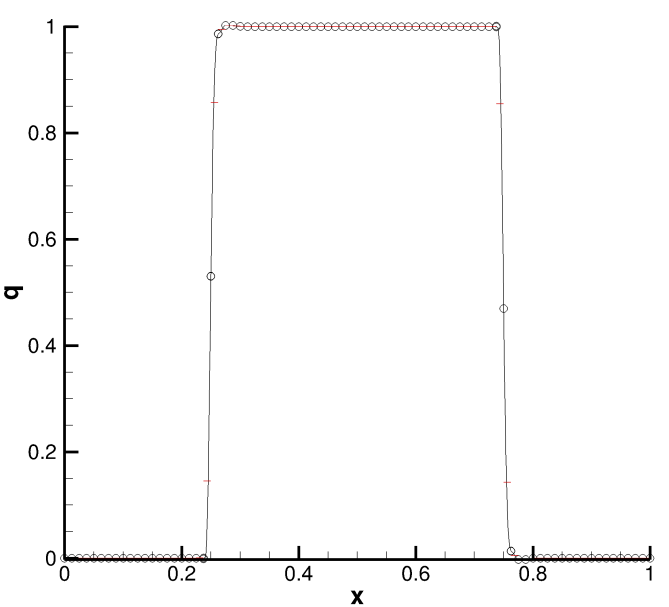

(b) DG-P5

Figure 5: Sub-cell distribution of square wave at $t=1.0$ with $P 1$-projected MLP.

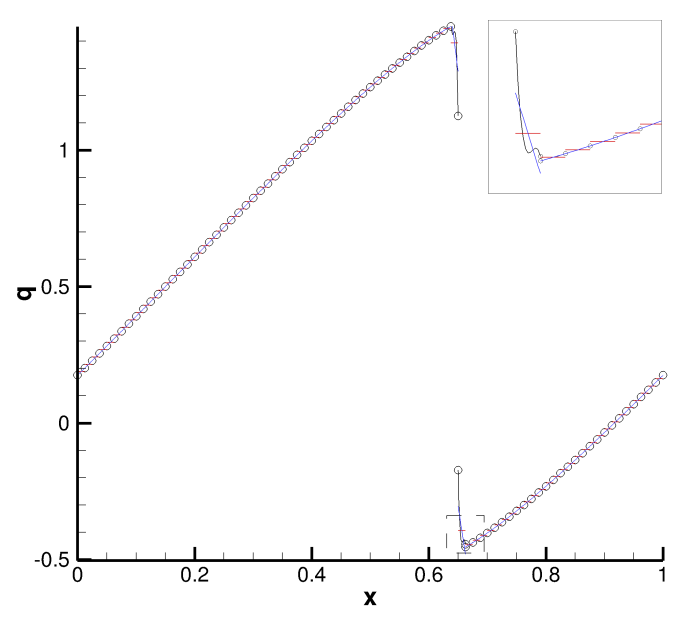

(a) DG-P3

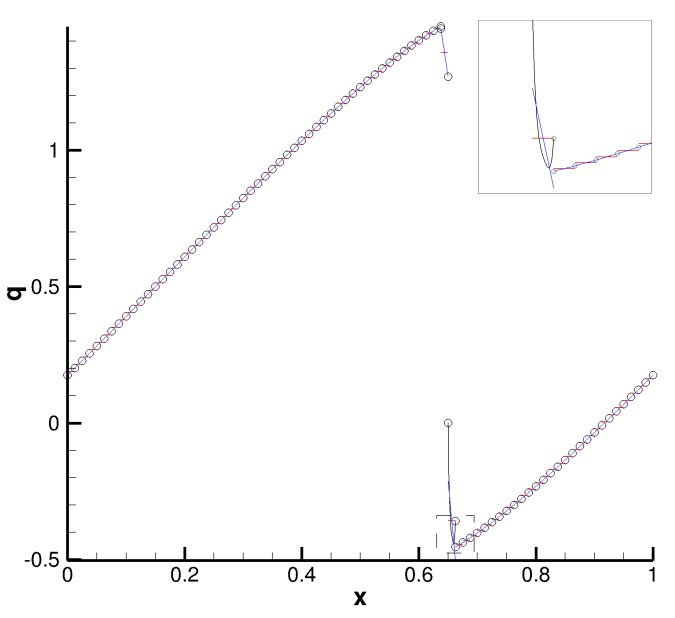

(b) DG-P4

Figure 6: Sub-cell distribution of Burgers' equation at $t=0.3$ with $P 1$-projected MLP. 
For GPU computing, CUDA programming is employed. GPU is a wide SIMD many-core architecture, thus parallel programing is carried out from a thread perspective. The main target of parallelization is Eq. (7), and two parallel loops are considered. First, the overall computation of $\mathbf{Q}_{i, j}^{h}$ can be executed independently, thus update procedure for each solution point is assigned to one thread. Second, the lifting operator $\delta_{i, j}$, the last term of Eq. (7) is computed by integrating it over the interfaces of the cell $T_{j}$ as Eqs. (8) and (9). Here, $\alpha_{l, f, j}$ and $[\mathbf{F}]_{l, f}$ is computed for each $l$-th solution point on the face $f$ with the same equations independent of $l$ and $f$. This leads to that each thread obtains and stores each $[\mathbf{F}]_{l, f}$ and, afterwards, it is summed to find out $\delta_{i, j}$ when updating $\mathbf{Q}_{i, j}^{h}$ of Eq. (7).

To implement the hierarchical MLP on GPU, $\bar{q}_{v_{i}}^{\max }$ and $\bar{q}_{v_{i}}^{\min }$ (Eq. (17)) are obtained in parallel by assigning a thread of GPU for each vertex $v_{i}$. The troubled-cell marking and limiting procedure for each cell is computed simultaneously on GPU. NVIDIA GTX 580 is used for GPU computation and computed results are compared with MPI parallel computation on two Xeon E5620 CPUs.

\section{Numerical Results}

Extensive numerical experiments are carried out to assess the performance of the hierarchical MLP for CPR method. Some well-known test problems on 2-D triangular grids are examined up to the $P 3$ accuracy. As a numerical flux, RoeM ${ }^{30}$ scheme and AUSMPW $+{ }^{31}$ scheme are adopted.

\section{A. Convergence Study}

Numerical accuracy in multi-dimensional smooth flows without shock wave is examined by considering an inviscid compressible flow on both CPU and GPU environments. The initial condition is set to be $\left(\rho_{0}, u_{0}, v_{0}, p_{0}\right)=(1+0.2 \sin (\pi(x+y)), 0.7,0.3,1)$, and the exact solution of density is $1+0.2 \sin (\pi(x+y-t))$. The computational domain is $[0,2] \times[0,2]$, and a periodic boundary condition is applied. The error between a numerical solution and the exact solution is measured by $L^{p}$ error with $p=\infty, 1$ and 2 norm. For $p \geq 1$,

$$
L^{p} \text { error }=\frac{1}{\sum_{j}\left|T_{j}\right|}\left(\sum_{j}\left|T_{j}\right| \sum_{i}^{n d o f}\left|q_{j}^{h}\left(\mathbf{x}_{i}\right)-q_{\text {exact }}\left(\mathbf{x}_{i}\right)\right|^{p}\right)^{1 / p},
$$

where $\left|T_{j}\right|$ is the area of cell $T_{j}$. $L^{\infty}$ error is computed using the maximum difference among the solution points. Table 1 shows the result of GPU computation and their deviation with that of CPU computation. It demonstrates that the MLP method maintain the desired accuracy and the deviation of both $L^{\infty}$ and $L^{2}$ is about machine zero, confirming that GPU computation practically coincides with CPU computation.

Table 1: Grid refinement test for Euler equations on irregular grids at $t=1.0$.

\begin{tabular}{cccccccc}
\hline & DOF & $L^{\infty}$ & Deviation & Order & $L^{2}$ & Deviation & Order \\
\hline & $898 \times 6$ & $5.7896 \mathrm{E}-04$ & $3.0870 \mathrm{E}-14$ & & $9.3711 \mathrm{E}-05$ & $2.5400 \mathrm{E}-16$ & \\
CPR-P2 & $3594 \times 6$ & $8.7863 \mathrm{E}-05$ & $6.7502 \mathrm{E}-14$ & 3.06 & $1.1323 \mathrm{E}-05$ & $1.3700 \mathrm{E}-16$ & 3.05 \\
MLP & $14412 \times 6$ & $1.1325 \mathrm{E}-05$ & $1.0525 \mathrm{E}-13$ & 3.03 & $1.3805 \mathrm{E}-06$ & $2.8780 \mathrm{E}-16$ & 3.03 \\
& $57364 \times 6$ & $1.4227 \mathrm{E}-06$ & $2.7500 \mathrm{E}-13$ & 3.00 & $1.7049 \mathrm{E}-07$ & $1.9907 \mathrm{E}-15$ & 3.03 \\
\hline & $898 \times 10$ & $2.7501 \mathrm{E}-05$ & $1.7246 \mathrm{E}-12$ & & $2.6460 \mathrm{E}-06$ & $2.5336 \mathrm{E}-13$ & \\
CPR-P3 & $3594 \times 10$ & $1.7044 \mathrm{E}-06$ & $7.0099 \mathrm{E}-13$ & 4.01 & $1.5299 \mathrm{E}-07$ & $8.4038 \mathrm{E}-15$ & 4.11 \\
MLP & $14412 \times 10$ & $1.1859 \mathrm{E}-07$ & $9.2903 \mathrm{E}-13$ & 3.84 & $9.3350 \mathrm{E}-09$ & $4.3016 \mathrm{E}-14$ & 4.03 \\
& $57364 \times 10$ & $8.1012 \mathrm{E}-09$ & $3.4284 \mathrm{E}-13$ & 3.89 & $5.8645 \mathrm{E}-10$ & $1.7589 \mathrm{E}-14$ & 4.01 \\
\hline
\end{tabular}

\section{B. Shock Tube Problems}

Some well-known one-dimensional shock tube problems are computed in a two-dimensional manner to examine the capability of resolving various linear and non-linear waves on unstructured grids. 


\section{Sod and Harte-Lax Problems}

This test is to examine the capability to resolve various linear and non-linear waves on unstructured grids. The computational domain is $[0,1] \times[0,0.01]$ with a triangulation of 101 vertices in the $x$-direction and 11 vertices in the $y$-direction. Two Riemann-type initial conditions are considered.

Sod problem:

$$
\begin{aligned}
& \left(\rho_{L}, u_{L}, v_{L}, p_{L}\right)=(1,0,0,1) \\
& \left(\rho_{R}, u_{R}, v_{R}, p_{R}\right)=(0.125,0,0,0.1) .
\end{aligned}
$$

Harten-Lax problem:

$$
\begin{aligned}
& \left(\rho_{L}, u_{L}, v_{L}, p_{L}\right)=(0.445,0.698,0,3.528) \\
& \left(\rho_{R}, u_{R}, v_{R}, p_{R}\right)=(0.5,0,0,0.571) .
\end{aligned}
$$

The interface is initially located at $x=0.5$ and RoeM scheme is applied.

Figures 7 and 8 show the density distributions along the $x$-axis at $t=0.2$ (the Sod problem) and $t=0.13$ (the Harten-Lax problem), respectively. Discontinuous profiles are successfully captured without spurious oscillations. Compared to the FVM with MLP solutions, the CPR with MLP methods exhibit a clear advantage in capturing contact discontinuites and expansion corners.
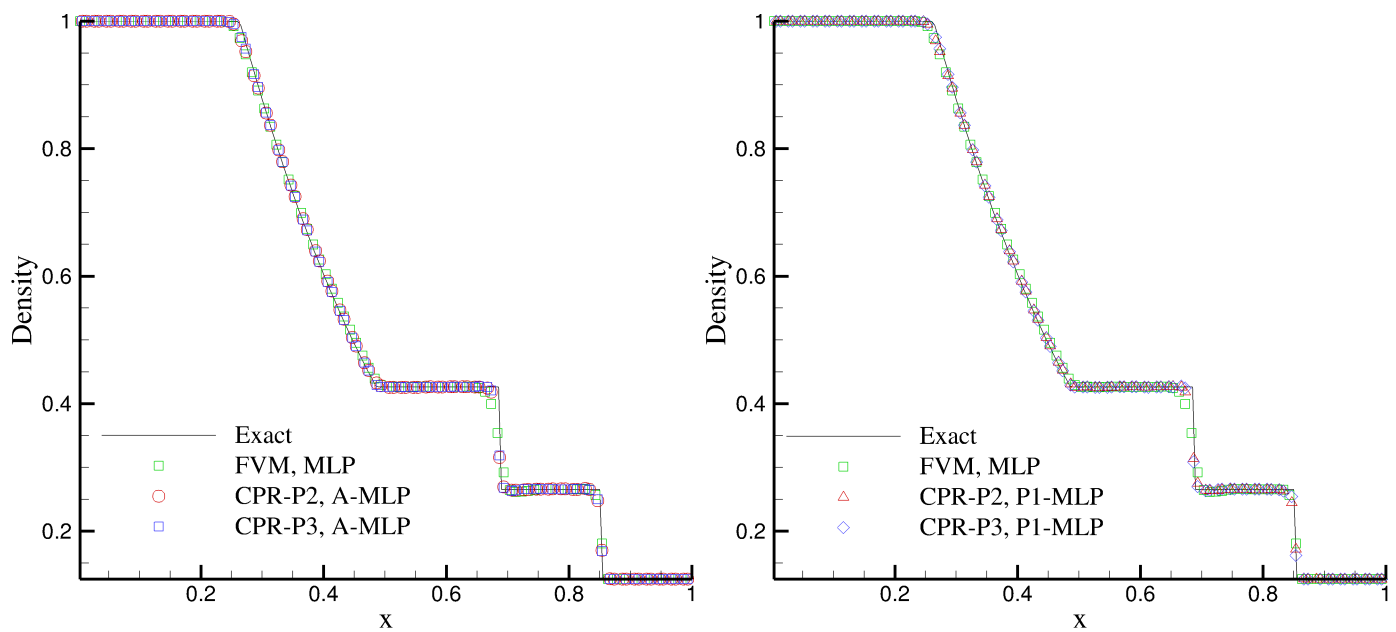

Figure 7: Density distributions along center line (Sod problem).
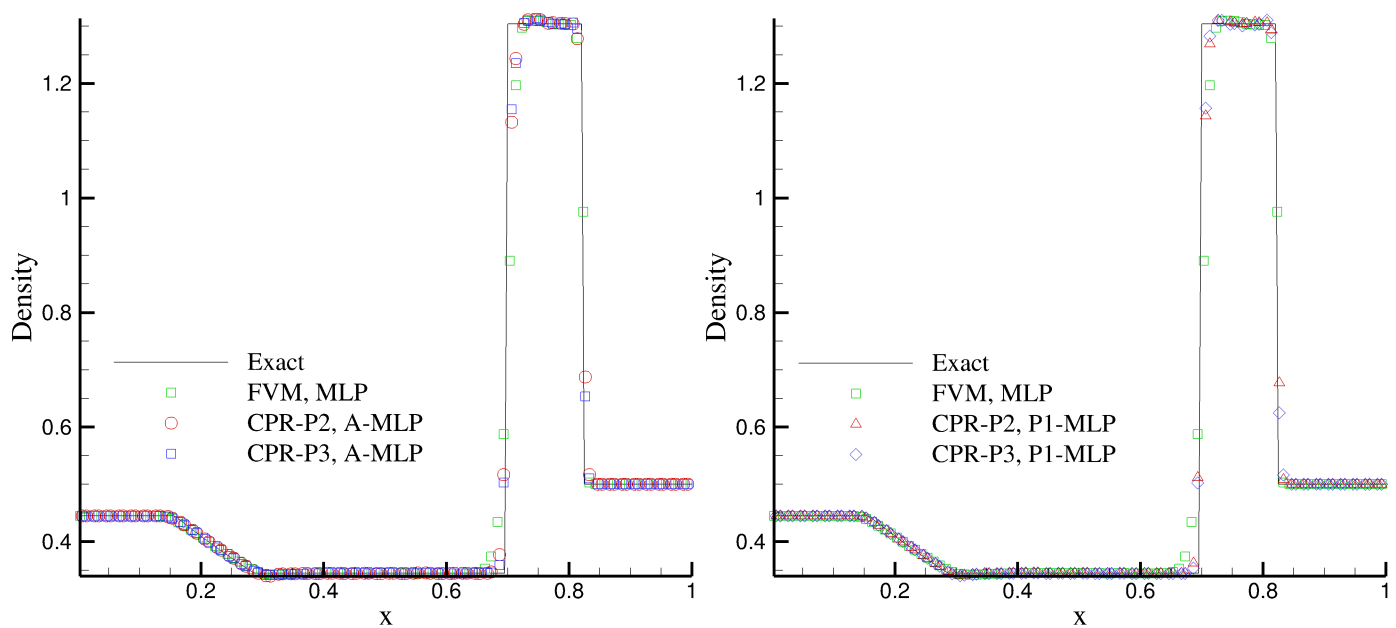

Figure 8: Density and internal energy distributions along center line (Harten-Lax problem). 


\section{Blast Wave Interaction}

In order to examine the robustness across strong shock waves, the interaction of blast waves ${ }^{32}$ is considered. Similar to the previous cases, a one-dimensional setting is extended into triangular grids. The computational domain is $[-5,5] \times[-0.1,0.1]$ with a triangulation of 301 vertices in the $x$-direction and 7 vertices in the $y$-direction. A reflective boundary condition is applied at both ends. The initial condition consists of the three constant states, as follows:

$$
\begin{array}{lll}
\left(\rho_{1}, u_{1}, v_{1}, p_{1}\right)=\left(1,0,0,1 \times 10^{3}\right) & \text { if } x \leq-4, \\
\left(\rho_{2}, u_{2}, v_{2}, p_{2}\right)=\left(1,0,0,1 \times 10^{-2}\right) & \text { if } x \leq 4, \\
\left(\rho_{3}, u_{3}, v_{3}, p_{3}\right)=\left(1,0,0,1 \times 10^{2}\right) & \text { else. }
\end{array}
$$

In Fig. 9, the computed density profile are compared at $t=0.38$. The reference solution is the one obtained by computing the equivalent one-dimensional Euler equations on 8,000 grid points using the TVDMUSCL scheme. The MLP limiting methods successfully resolve strong blast waves without yielding spurious oscillations, and the higher-order CPR-MLP methods capture the wave interaction more accurately.

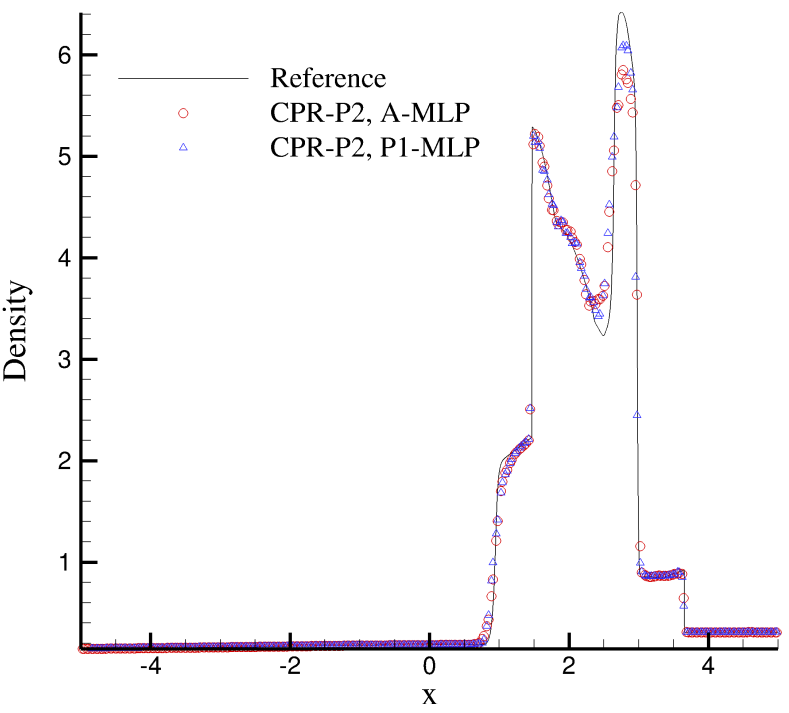

(a) CPR-P2

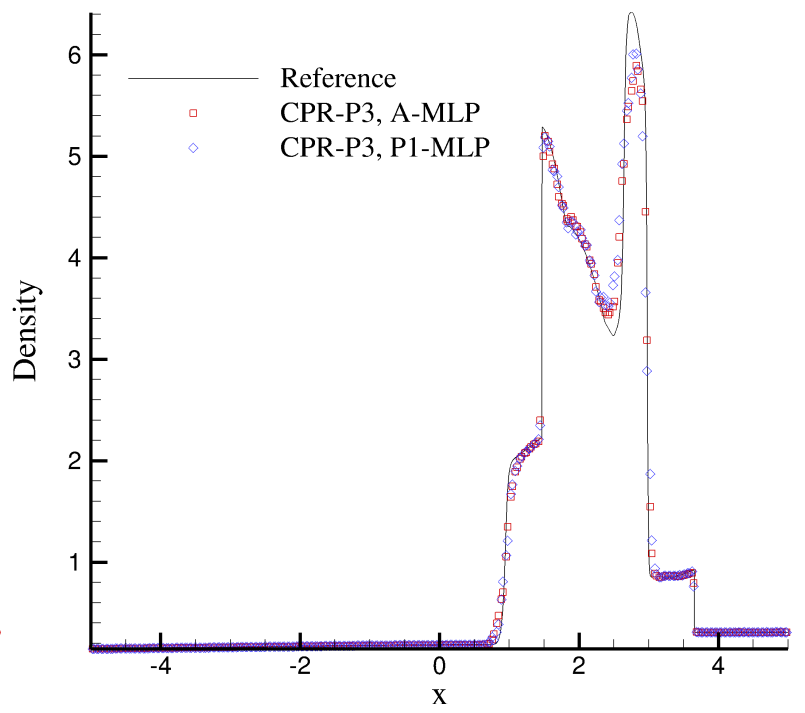

(b) CPR-P3

Figure 9: Density distributions along center line (Two blast-wave interaction).

\section{Shock-entropy Wave Interaction (Shu-Osher Problem)}

As another standard benchmark test for high-resolution schemes, the interaction between a shock and an entropy wave ${ }^{33}$ is considered. The computational domain and grid are the identical to those used in the previous case. The initial profile consists of a shock and an entropy wave, as follows:

$$
\begin{array}{ccl}
\left(\rho_{L}, u_{L}, v_{L}, p_{L}\right) & =(3.857143,2.629369,0,10.333333) & \text { if } x<-4, \\
\left(\rho_{R}, u_{R}, v_{R}, p_{R}\right) & =(1+0.2 \sin (5 x), 0,0,1.0) & \text { else. }
\end{array}
$$

Figure 10 compares the density distributions along the center line by CPR-P2 and CPR-P3 with MLP limiting at $t=1.8$. The reference solution is the one obtained by computing the equivalent one-dimensional Euler equations on 8,000 grid points using the TVD-MUSCL scheme. It is noted again that the MLP limiting, successfully combined with the CPR method, accurately suppresses unwanted oscillations. In particular, the hierarchical limiting with the $P 1$-projected MLP condition resolves the interaction more clearly.

\section{Double Mach reflection}

This is one of the most well-known test cases for high-resolution schemes. ${ }^{32}$ With the computational domain of a tube with a 30 degree ramp, a strong moving shock with $M_{s}=10$ impinges on the ramp. AUSMPW+ scheme is used as a numerical flux, and computation is carried out until $t=0.2$. 


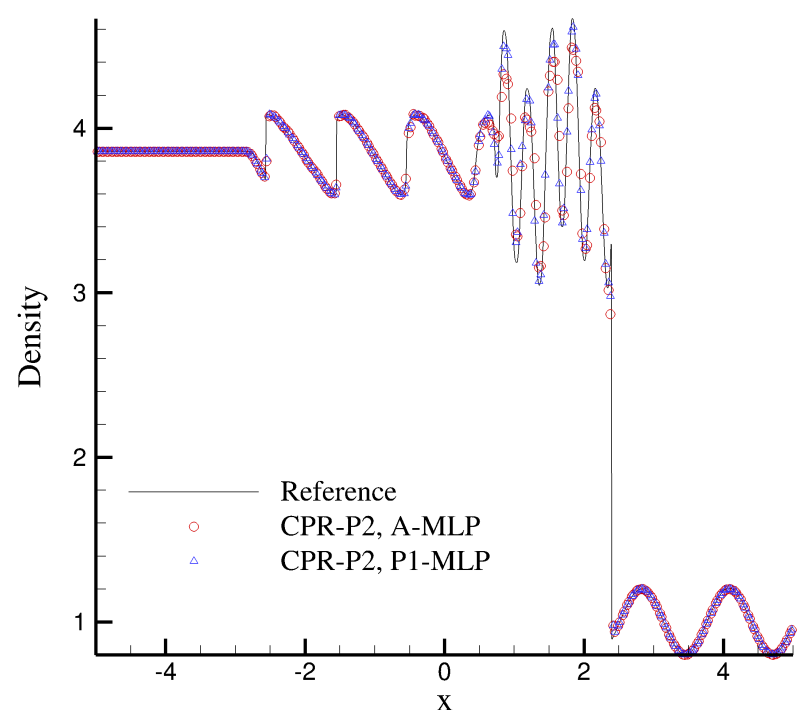

(a) CPR-P2 with MLP

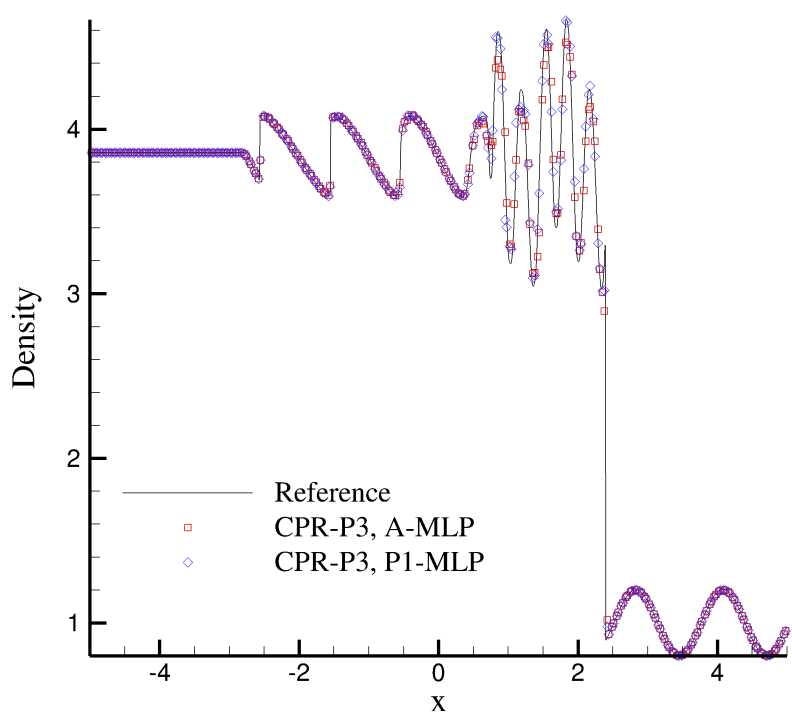

(b) CPR-P3 with MLP

Figure 10: Density distributions along center line (Shock-entropy wave Interaction).

Figure 11 shows the comparison of density contours with CPR-MLP methods on triangular grid ( $h=$ 1/100). The MLP limiting successfully provide monotonic solutions. The higher-order CPR methods significantly improve the resolution of the sheer layer and vortex developed from the shock triple point and the Mach stem.

Table 2 shows the relative computational costs for MLP methods. Due to simplicity by nodal formulation, CPR requires about $70 \sim 75 \%$ cost of DG method. In addition, GPU computation on GTX580 is about 10 $\sim 30 \%$ faster than CPU parallel computation on two Xeon processor.

Table 2: Comparison of relative computational costs

\begin{tabular}{cccc}
\hline & \multicolumn{2}{c}{ CPU } & GPU \\
& DG & CPR & CPR \\
\hline P2 & 1.38 & 1.0 & 0.74 \\
P3 & 3.21 & 2.36 & 2.15 \\
\hline
\end{tabular}

\section{A Mach 3 wind tunnel with a step}

This is another standard test case to examine high-resolution schemes. ${ }^{32}$ The problem begins with a uniform Mach 3 flow in a wind tunnel with a step, whose size is 3 length unit long and 1 length unit high. The step is 0.2 length unit high, located at 0.6 length unit from the left end of the tunnel. Reflective boundary condition is applied along the wall of the tunnel, and in-flow free stream and out-flow extrapolation conditions are applied at the entrance and the exit. Around the expansion corner, computational meshes are slightly clustered without any special treatment, as in Ref. ${ }^{10}$ RoeM scheme is applied as a numerical flux.

Figure 12 shows the density contours computed on triangular grids of $h=1 / 160$ at $t=4.0$. Similar to the previous test case, one can check the resolving capability of the MLP methods for the shear layer instability from the shock triple point. Table 3 shows again that GPU computation provides a better performance.

\section{E. Interaction of Shock Wave with 2-D Wedge}

This test case is known as the Scharldin's problem. ${ }^{34}$ As a moving shock strikes a two-dimensional wedge, reflected shock waves and complex vortex pattern are generated after the wedge end. A regular triangle with the unit length is contained in the computational domain of $[-2.5,4.6] \times[-2.5,2.5]$, and the wedge tip is 


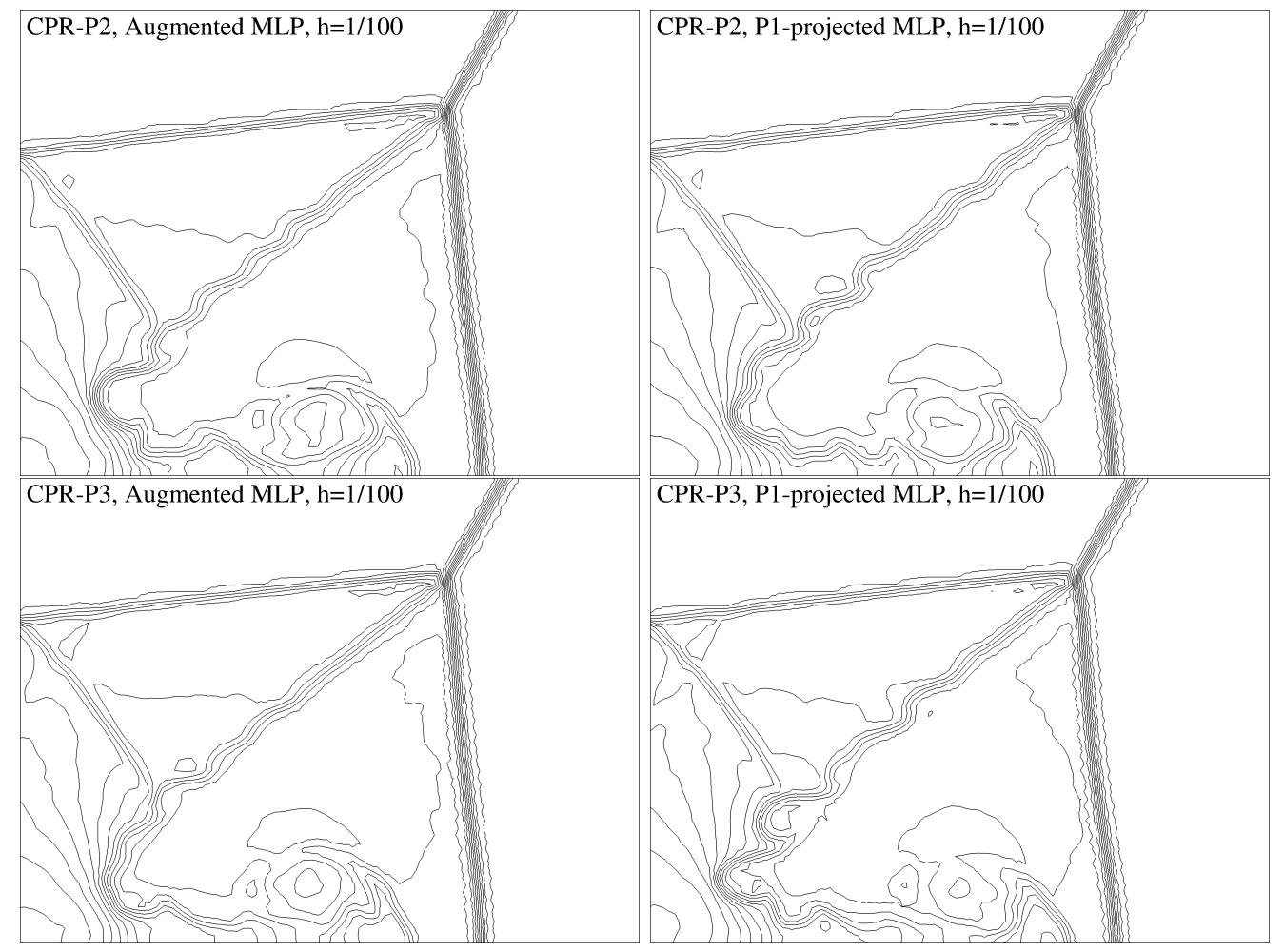

Figure 11: Comparison of density contours around the double Mach stem (CPR-MLP).

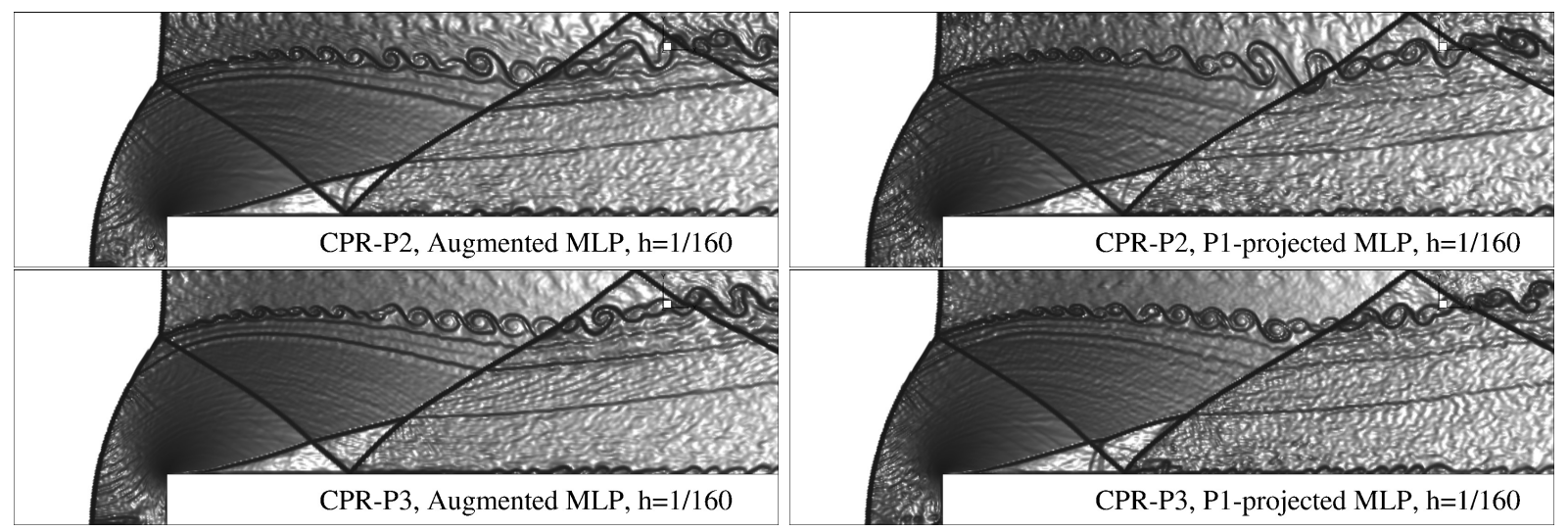

Figure 12: Comparison of density contours for the Mach 3 wind tunnel with a step (CPR-MLP).

Table 3: Comparison of relative computational costs

\begin{tabular}{ccc}
\hline & CPU & GPU \\
\hline CPR-P2 & 1.0 & 0.70 \\
CPR-P3 & 2.30 & 2.03 \\
\hline
\end{tabular}


positioned at the origin. As the initial condition, a moving shock with $M_{s}=1.34$ is located at $x=0$. The downstream state is $\left(\rho_{0}, u_{0}, v_{0}, p_{0}\right)=(1.4,0,0,1.0)$. The RoeM flux scheme is applied.

Figure 13 compares the numerical Schlieren images produced by the FVM, DG- $P 1$ and CPR with MLP at $t=3.25$. The computed results confirm again that the higher-order CPR with the MLP limiting provides a resolution sufficient to capture the detailed shock pattern and flow structures. Compared with experimental visualization, ${ }^{35}$ the small vortices produced by the first shock-vortex interaction at the wedge corner are particularly well captured by the hierarchical limiting with the P1-projected MLP condition.

\section{F. Oblique Shock Mixing Layer Interaction}

This test is done to compute a small scale vortical structure interacting with a shock discontinuity. ${ }^{36}$ A spatially developing mixing layer produces a series of vortices, and the oblique shock originating from the upper-left corner impinges on the mixing layer. The oblique shock is deflected by the shear layer and then reflects again from the bottom slip wall, leading to the interaction between downstream vortices and the reflected shock.

For the initial condition, a hyperbolic tangent velocity profile and a convective Mach number are imposed.

$$
\begin{gathered}
u=2.5+0.5 \tanh (2 y), \\
M_{c}=\frac{u_{1}-u_{2}}{c_{1}-c_{2}}=0.6 .
\end{gathered}
$$

The upper boundary condition is specified to impose the oblique shock with a shock-angle of $\beta=12^{\circ}$, and the lower boundary condition is a slip wall. Fluctuation adding to the mean in-flow is given by

$$
v^{\prime}=\sum_{k=1}^{2} a_{k} \cos \left(2 \pi k t / T+\phi_{k}\right) \exp \left(-y^{2} / b\right),
$$

with a period $T=\lambda / u_{c}$, a wavelength $\lambda=30$ and a convective velocity $u_{c}=2.68$. The other parameters are as follows: $a_{1}=a_{2}=0.05, \phi_{1}=0, \phi=\pi / 2$ and $b=10$. The Reynolds number and the Prandtl number are 500 and 0.72 , respectively. The computational domain is $[0,200] \times[-20,20]$. For a better resolution, many filter methods have computed this problem on meshes clustered along the $y$-direction, but the present computation employs uniformly distributed triangular grids of $h=0.75$. As a numerical flux, the RoeM scheme is used.

Figures 14 show a comparison of the pressure contours using the FVM and CPR with MLP limitings at $t=120$. While the vortex structure by the second-order FVM with MLP on a coarse grid is mostly smeared out, the higher-order CPR method with MLP resolve it more clearly. The result of the FVM with MLP method on fine grid $(h=0.5)$ is even diffusive than CPR-P2 on coarse grid. The CPR-P3 with MLP on fine grid provides the most detailed flow structure, especially for the downstream shock-vortex interaction.

\section{Conclusions}

Guided by the MLP condition and the maximum principle, the hierarchical MLP limiting is successfully extended into the higher-order CPR method, as well as the DG method. The augmented MLP condition and $P 1$-projected MLP condition are proposed to treat solution points near discontinuities. The uncertainty of determining a parameter for the slope limiting is then eliminated by examining the behavior of local extrema near vertex point, which leads to the formulation of the hierarchical MLP limiting.

Extensive computations are carried out up to $P 3$ approximation to examine the capability of the hierarchical MLP methods in capturing multi-dimensional flow physics. Numerous comparisons on unstructured grids demonstrate the proposed limiting provides detailed multi-dimensional flow structures without numerical oscillations in discontinuous region, while maintaining the required accuracy in smooth region. Both CPU and GPU parallel computations are carried out and GPU computation on one GTX580 outperforms MPI parallel computation on two quad-core Xeon processor.

\section{Acknowledgments}

Authors appreciate the financial supports provided by NSL (National Space Lab.) program through the National Research Foundation of Korea funded by the Ministry of Education, Science and Technology 


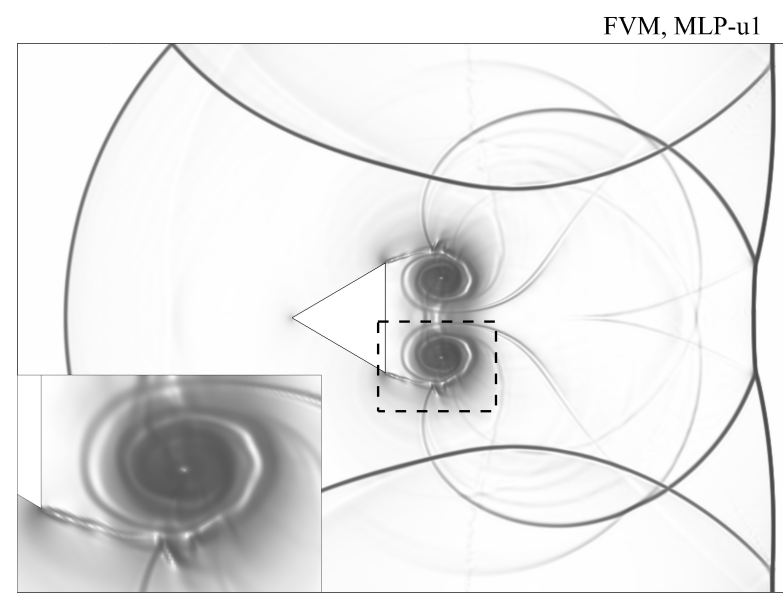

(a) FVM

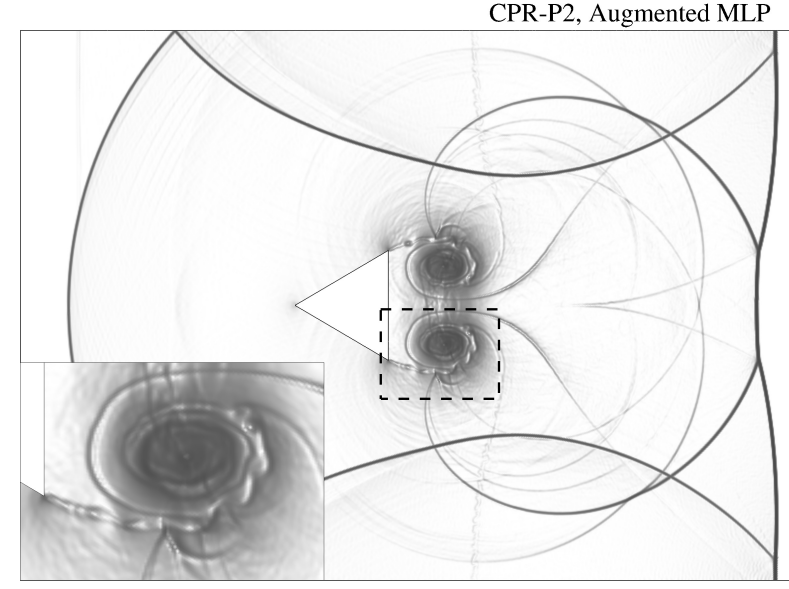

(c) CPR-P2, Hierarchical MLP

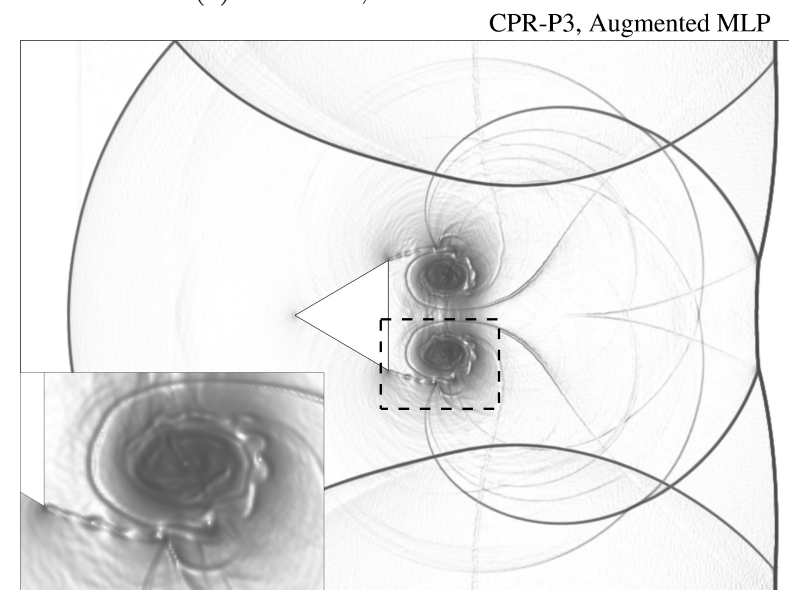

(e) CPR-P3, Hierarchical MLP

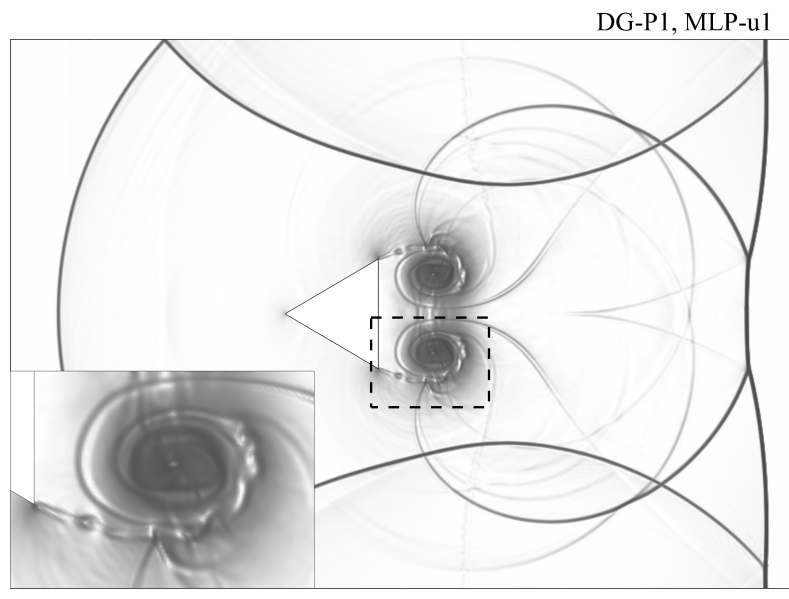

(b) DG- $P 1$

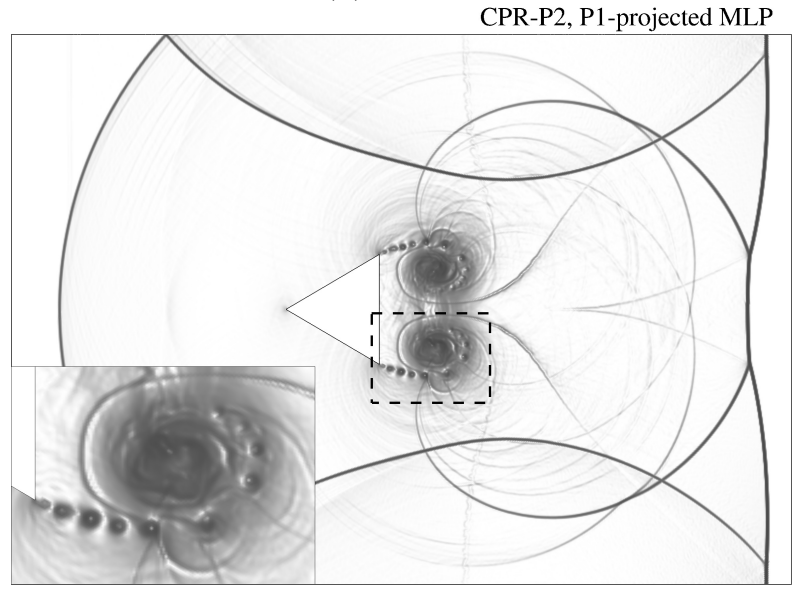

(d) CPR-P2, projected-P1 MLP

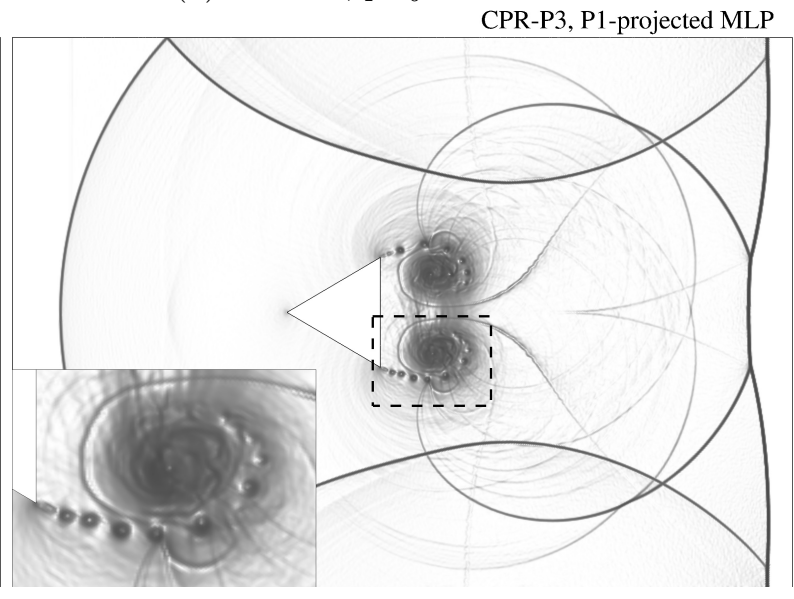

(f) CPR-P3, projected-P1 MLP

Figure 13: Comparison of numerical Schlieren images of interaction of shock wave with 2-D wedge at $t=3.25$ by CPR-MLP. (Bottom left corner: Close-up view around the primary vortex) 


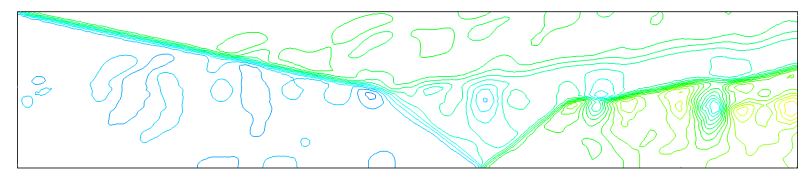

(a) Pressure, FVM

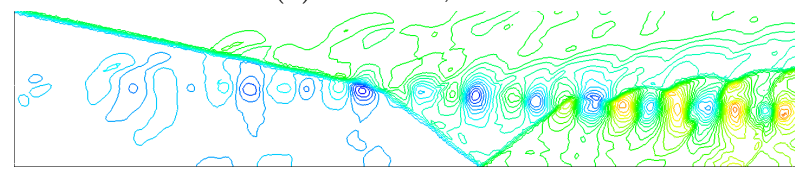

(c) Pressure, CPR-P2,Augmented MLP

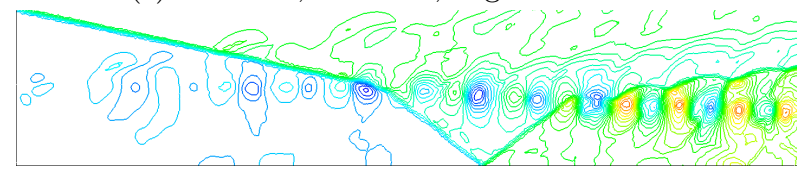

(e) Pressure, CPR-P3, Augmented MLP

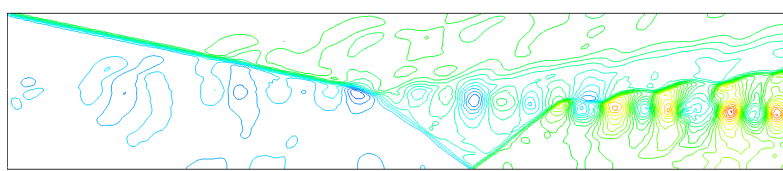

(b) Pressure, FVM (fine grid)

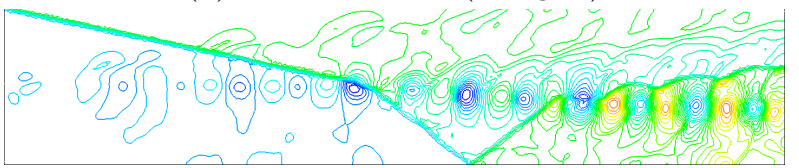

(d) Pressure, CPR-P2, P1-projected MLP

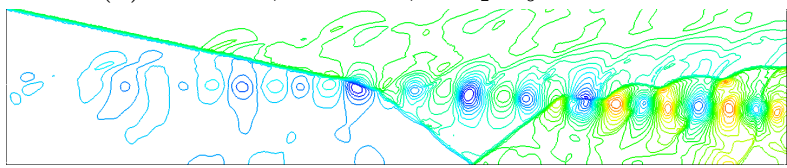

(f) Pressure, CPR-P3, P1-projected MLP

Figure 14: Comparison of pressure contours of shock-mixing layer interaction on coarse grid

(Grant 20090091724), by the third stage of the Brain Korea 21 Plus Project in 2013, and by Korea Ministry of Land, Transport and Maritime Affairs as Haneul Project. This work is also supported by the KISTI Supercomputing Center (KSC-2012-C3-40) and by PLSI supercomputing resources of KISTI.

\section{References}

${ }^{1}$ Cockburn, B., Karniadakis, G. E., and Shu, C.-W., Discontinuous Galerkin Methods: Theory, Computation and Applications, Springer-Verlag Inc., 2000.

${ }^{2}$ Wang, Z. J., "High-order methods for the Euler and NavierStokes equations on unstructured grids," Progress in Aerospace Sciences, Vol. 43, No. 1-3, Jan. 2007, pp. 1-41.

${ }^{3}$ Wang, Z. J., Adaptive High-Order Methods in Computational Fluid Dynamics, Vol. 2, World Scientific Pub Co Inc, 2011.

${ }^{4}$ Wang, Z. J., "Spectral (Finite) volume method for conservation laws on unstructured grids: basic formulation," Journal of Computational Physics, Vol. 178, No. 1, 2002, pp. 210-251.

${ }^{5}$ Wang, Z. J., Liu, Y., May, G., and Jameson, A., "Spectral difference method for unstructured grids II: Extension to the Euler equations," Journal of Scientific Computing, Vol. 32, No. 1, 2007, pp. 45-71.

${ }^{6}$ Huynh, H. T., "A flux reconstruction approach to high-order schemes including discontinuous Galerkin methods," 18th AIAA Computational Fluid Dynamics Conference, Miami, FL, 2007.

${ }^{7}$ Huynh, H. T., "A reconstruction approach to high-order schemes including discontinuous Galerkin for diffusion," $47 t h$ AIAA Aerospace Sciences Meeting Including The New Horizons Forum and Aerospace Exposition, Orlando, FL, 2009.

${ }^{8}$ Wang, Z. J. and Gao, H., "A unifying lifting collocation penalty formulation including the discontinuous Galerkin, spectral volume/difference methods for conservation laws on mixed grids," Journal of Computational Physics, Vol. 228, No. 21, Nov. 2009, pp. 8161-8186.

${ }^{9}$ Cook, A. W. and Cabot, W. H., "Hyperviscosity for shock-turbulence interactions," Journal of Computational Physics, Vol. 203, No. 2, 2005, pp. 379-385.

${ }^{10}$ Cockburn, B. and Shu, C.-W., "The RungeKutta discontinuous Galerkin method for conservation laws V: multidimensional systems," Journal of Computational Physics, Vol. 141, 1998, pp. 199-224.

${ }^{11}$ Biswas, R., Devine, K. D., and Flaherty, J. E., "Parallel, adaptive finite element methods for conservation laws," Applied Numerical Mathematics, Vol. 14, No. 1-3, 1994, pp. 255-283.

${ }^{12}$ Burbeau, A., Sagaut, P., and Bruneau, C.-H., "A problem-independent limiter for high-order RungeKutta discontinuous Galerkin methods," Journal of Computational Physics, Vol. 169, No. 1, May 2001, pp. 111-150.

${ }^{13}$ Krivodonova, L., "Limiters for high-order discontinuous Galerkin methods," Journal of Computational Physics, Vol. 226, No. 1, Sept. 2007, pp. 879-896.

${ }^{14}$ Yang, M. and Wang, Z. J., "A parameter-free generalized moment limiter for high-order methods on unstructured grids," Advances in Applied Mathematics and Mechanics, No. January, 2009, pp. 1-22.

${ }^{15} \mathrm{Xu}, \mathrm{Z}$., Liu, Y., and Shu, C.-W., "Hierarchical reconstruction for discontinuous Galerkin methods on unstructured grids with a WENO-type linear reconstruction and partial neighboring cells," Journal of Computational Physics, Vol. 228, No. 6, April 2009, pp. 2194-2212.

${ }^{16}$ Xu, Z., Liu, Y., Du, H., Lin, G., and Shu, C.-W., "Point-wise hierarchical reconstruction for discontinuous Galerkin and finite volume methods for solving conservation laws," Journal of Computational Physics, Vol. 230, No. 17, July 2011, pp. 6843-6865.

${ }^{17}$ Zhu, J., Qiu, J., Shu, C.-W., and Dumbser, M., "RungeKutta discontinuous Galerkin method using WENO limiters II: Unstructured meshes," Journal of Computational Physics, Vol. 227, No. 9, April 2008, pp. 4330-4353. 
${ }^{18}$ Luo, H., Baum, J. D., and Löhner, R., "A discontinuous Galerkin method based on a Taylor basis for the compressible flows on arbitrary grids," Journal of Computational Physics, Vol. 227, No. 20, Oct. 2008, pp. 8875-8893.

${ }^{19} \mathrm{Zhu}$, J. and Qiu, J., "Hermite WENO Schemes and Their Application as Limiters for Runge-Kutta Discontinuous Galerkin Method, III: Unstructured Meshes," Journal of Scientific Computing, Vol. 39, No. 2, Jan. 2009, pp. $293-321$.

${ }^{20} \mathrm{Kim}, \mathrm{K} . \mathrm{H}$. and Kim, C., "Accurate, efficient and monotonic numerical methods for multi-dimensional compressible flows Part II: Multi-dimensional limiting process," Journal of Computational Physics, Vol. 208, No. 2, Sept. 2005, pp. 570-615.

${ }^{21}$ Park, J. S., Yoon, S.-H., and Kim, C., "Multi-dimensional limiting process for hyperbolic conservation laws on unstructured grids," Journal of Computational Physics, Vol. 229, No. 3, Feb. 2010, pp. 788-812.

${ }^{22}$ Park, J. S. and Kim, C., "Multi-dimensional limiting process for finite volume methods on unstructured grids," Computers E Fluids, Vol. 65, No. 0, July 2012, pp. 8-24.

${ }^{23}$ Park, J. S. and Kim, C., "Higher-order multi-dimensional limiting strategy for discontinuous Galerkin methods in compressible inviscid and viscous flows," Computers 8 Fluids, Vol. In press.

${ }^{24} \mathrm{Gao}, \mathrm{H}$. and Wang, Z. J., "A conservative correction procedure via reconstruction formulation with the Chain-Rule divergence evaluation," Journal of Computational Physics, Vol. 232, No. 1, Jan. 2013, pp. 7-13.

${ }^{25}$ Gao, H., Wang, Z. J., and Huynh, H. T., "Differential formulation of discontinuous Galerkin and related methods for the Navier-Stokes equations," Communications in Computational Physics, Vol. 13, No. 4, 2012, pp. 1013-1044.

${ }^{26}$ Bassi, F., Crivellini, A., Rebay, S., and Savini, M., "Discontinuous Galerkin solution of the Reynolds-averaged NavierStokes and k-w turbulence model equations," Computers 83 Fluids, Vol. 34, No. 4?5, 2005, pp. 507-540.

${ }^{27}$ Spiteri, R. J. and Ruuth, S. J., "A new class of optimal high-order strong-stability-preserving time discretization methods," SIAM Journal on Numerical Analysis, Vol. 40, No. 2, 2001, pp. 469-491.

${ }^{28}$ Krivodonova, L., Xin, J., Remacle, J.-F., Chevaugeon, N., and Flaherty, J. E., "Shock detection and limiting with discontinuous Galerkin methods for hyperbolic conservation laws," Applied Numerical Mathematics, Vol. 48, No. 3-4, March 2004, pp. 323-338.

${ }^{29}$ Karypis, G. and Kumar, V., "Multilevel k-way partitioning scheme for irregular graphs,," Journal of Parallel and Distributed Computing, Vol. 48, No. 1, 1998, pp. 96-129.

${ }^{30}$ Kim, S.-s., Kim, C., Rho, O.-H., and Hong, S. K., "Cures for the shock instability: Development of a shock-stable Roe scheme," Journal of Computational Physics, Vol. 185, No. 2, March 2003, pp. 342-374.

${ }^{31} \mathrm{Kim}, \mathrm{K} . \mathrm{H}$., Kim, C., and Rho, O.-H., "Methods for the accurate computations of hypersonic flows I. AUSMPW+ scheme," Journal of Computational Physics, Vol. 174, No. 1, Nov. 2001, pp. 38-80.

${ }^{32}$ Woodward, P. R. and Colella, P., "The numerical simulation of two-dimensional fluid flow with strong shocks," Journal of Computational Physics, Vol. 54, No. 1, 1984, pp. 115-173.

${ }^{33} \mathrm{Shu}$, C.-W. and Osher, S., "Efficient implementation of essentially non-oscillatory shock-capturing schemes, II," Journal of Computational Physics, Vol. 83, No. 1, 1989, pp. 32-78.

${ }^{34}$ Dyke, M. V., An album of fluid motion, The Parabolic Press, 1982.

${ }^{35}$ Chang, S.-M. and Chang, K.-S., "On the shock-vortex interaction in Schardin's problem," Shock Waves, Vol. 10, No. 5, Nov. 2000, pp. 333-343.

${ }^{36}$ Yee, H. C., Sandham, N. D., and Djomehri, M. J., "Low-dissipative high-order shock-capturing methods using characteristic-based filters," Journal of Computational Physics, Vol. 150, No. 1, 1999, pp. 199-238. 